\title{
Zero dissipation limit of full compressible Navier-Stokes equations with a Riemann initial data
}

\author{
FEIMIN HUANG* ${ }^{*}$ SONG JIANG ${ }^{\dagger}$, AND Yi WANG ${ }^{\ddagger}$ \\ Dedicated to Professor Marshall Slemrod on the occasion \\ of his 70th birthday
}

\begin{abstract}
We consider the zero dissipation limit of the full compressible Navier-Stokes equations with a Riemann initial data for the superposition of two rarefaction waves and a contact discontinuity. It is proved that for any suitably small viscosity $\varepsilon$ and heat conductivity $\kappa$ satisfying the relation (1.3), there exists a unique global piecewise smooth solution to the compressible Navier-Stokes equations. Moreover, as the viscosity $\varepsilon$ tends to zero, the Navier-Stokes solution converges uniformly to the Riemann solution of superposition of two rarefaction waves and a contact discontinuity to the corresponding Euler equations with the same Riemann initial data away from the initial line $t=0$ and the contact discontinuity located at $x=0$.
\end{abstract}

\section{Introduction}

We study the zero dissipation limit of the solution to the Navier-Stokes equations of a compressible heat-conducting gas in Lagrangian coordinate:

$$
\left\{\begin{array}{l}
v_{t}-u_{x}=0 \\
u_{t}+p_{x}=\varepsilon\left(\frac{u_{x}}{v}\right)_{x}, \\
\left(e+\frac{u^{2}}{2}\right)_{t}+(p u)_{x}=\kappa\left(\frac{\theta_{x}}{v}\right)_{x}+\varepsilon\left(\frac{u u_{x}}{v}\right)_{x}
\end{array}\right.
$$

${ }^{*}$ F. Huang is supported was supported in part by NSFC Grant No. 11371349, National Basic Research Program of China (973 Program) under Grant No. 2011CB808002, and the CAS Program for Cross \& Cooperative Team of the Science \& Technology Innovation.

${ }^{\dagger} \mathrm{S}$. Jiang is supported in part by the National Basic Research Program (Grant No. 2011CB309705, 2014CB745000), NSFC (Grant No. 11229101, 11371065).

$\ddagger$ Corresponding author. Y. Wang is supported by NSFC (Grant No. 11322106 and No. 11171326). 
with Riemann initial data

$$
(v, u, \theta)(0, x)= \begin{cases}\left(v_{-}, u_{-}, \theta_{-}\right), & x<0 \\ \left(v_{+}, u_{+}, \theta_{+}\right), & x>0\end{cases}
$$

where the functions $v(x, t)>0, u(x, t), \theta(x, t)>0$ represent the specific volume, velocity and the absolute temperature of the gas, respectively. And $p=p(v, \theta)$ is the pressure, $e=e(v, \theta)$ is the internal energy, $\varepsilon>0$ is the viscosity constant and $\kappa>0$ is the coefficient of heat conduction. Here we consider an ideal and polytropic gas, that is

$$
p=\frac{R \theta}{v}, \quad e=\frac{R \theta}{\gamma-1},
$$

with $\gamma>1, R>0$ being gas constants.

The study of the asymptotic behavior of viscous flows, as the viscosity tends to zero, is one of the important problems in the theory of compressible fluid flows. When the solution of the inviscid flow is smooth, the zero dissipation limit problem can be solved by classical scaling method. However, the inviscid compressible flow contains discontinuities, such as shock waves, in general. In this case, it is also conjectured that a general weak entropy solution to the inviscid flow should be the strong limit of the solution to the corresponding viscous flows with the same initial data as the viscosity vanishes.

It is well known that the solution to the Riemann problem for the Euler equations consists of three basic wave patterns, that is, shock, rarefaction wave and contact discontinuity. Moreover, the Riemann solution is essential in the theory for the Euler equations as it captures both local and global behavior of general solutions.

For hyperbolic conservation laws with the uniform viscosity

$$
u_{t}+f(u)_{x}=\varepsilon u_{x x},
$$

where $f(u)$ satisfies some assumptions to ensure the hyperbolic nature of the corresponding inviscid system, Goodman-Xin [4] verified the limit for piecewise smooth solutions separated by non-interacting shock waves using a matched asymptotic expansion method. Later, Yu [30] proved it for hyperbolic conservation laws with both shock and initial layers. In 2005, important progress made by Bianchini-Bressan [1] justifies the vanishing viscosity limit in BV-space even though the problem is still unsolved for the physical system such as the compressible Navier-Stokes equations. For the multidimensional case, the existence of curved, multidimensional viscous shocks to the viscous conservation laws and the small-viscosity limit to the corresponding hyper- 
bolic conservation laws were proved by Guès-Métivier-Williams-Zumbrun in $[5,6]$.

For the compressible isentropic Navier-Stokes equations where the conservation of energy in (1.1) is neglected in the isentropic regime, Hoff-Liu [13] firstly proved the vanishing viscosity limit for a piecewise constant shock with initial layer. Later, Xin [28] justified the limit for rarefaction waves and Wang [26] generalized the result of Goodman-Xin [4] to the isentropic Navier-Stokes equations. Then, Huang-Li-Wang [14] investigated the zero dissipation limit of the one-dimensional compressible isentropic NavierStokes equations in the case of rarefaction wave connected with one-side vacuum state.

Recently, Chen-Perepelitsa [2] proved the convergence of the isentropic compressible Navier-Stokes equations to the compressible Euler equations as the viscosity vanishes in Eulerian coordinates for general initial data by using compensated compactness method if the far field does not contain vacuum. Note that this result allows the initial data containing vacuum in the interior domain. However, the framework of compensated compactness is basically limited to $2 \times 2$ systems so far, so that this result could not be applied to the full compressible Navier-Stokes equations (1.1).

For the full compressible Navier-Stokes equations, there are investigations on the limits to the Euler system for the basic wave patterns in the literature. We refer to Jiang-Ni-Sun [20] and Xin-Zeng [29] for the rarefaction wave, Wang [27] for the single shock wave, Zhang-Pan-Wang-Tan [31] for the two shock waves, Ma [21] for the contact discontinuity and HuangWang-Yang $[17,18]$ for the superposition of two rarefaction waves and a contact discontinuity and the superposition of rarefaction and shock waves, respectively. Recently, Huang-Wang-Wang-Yang [19] justified the vanishing viscosity limit of 1D full compressible Navier-Stokes equations to the Euler equations for generic Riemann solution with the superposition of shock, rarefaction waves and contact discontinuity. We should point out that the limits shown in [20] and [31] are for the discontinuous initial data while the other results mentioned are for (well-prepared) smooth data.

As mentioned above, for the zero dissipation limit of the full NavierStokes equations (1.1) with discontinous Riemann initial data (1.2), the only known results are Jiang-Ni-Sun [20] for the rarefaction waves case and Zhang-Pan-Wang-Tan [31] for the two shock waves case. In this paper, we shall investigate the case of different wave patterns, that is, the superposition of two rarefaction waves and a contact discontinuity. The local and global well-posedness of the full system (1.1) or the corresponding isentropic system with discontinuous initial data is systematically studied by Hoff, etc., see 
$[7,8,9,10,11,12,3]$. In order to get the zero dissipation limit of the system (1.1) to the Riemann solution of the Euler system, on one hand, we shall combine the local existence of solutions with discontinuous data from [9] and the time-asymptotic stability analysis to the compressible Navier-Stokes equations (2.2); on the other hand, one must take care of the propagation of the discontinuity uniformly in time. Compared with the previous results in [17] where the same limit process is studied for (well-prepared) smooth initial data and in [15] for the stability of two rarefaction waves and a contact discontinuity, the main difficulty here lies in the propagation of the discontinuity from the initial data. The discontinuity of the initial data for the volume $v(t, x)$ will propagate for all the time along the particle path due to the hyperbolic regime while the smoothing effects will also be performed on the velocity $u(t, x)$ and the temperature $\theta(t, x)$ by the parabolic structure, and this interaction of the discontinuity and smoothing effects brings technical difficulties. On the other hand, compared with $[20,31]$ where the zero dissipation limit is shown for the rarefaction or two shock waves case, the difficulties here arise from the contact wave and its interactions with the rarefaction waves. To circumvent such difficulties, motivated by Huang-Li-Matsumura [15] for the stability of two rarefaction waves and a contact discontinuity in the middle, we shall choose suitable weight functions to carry out the weighted energy estimates in terms of the superposition wave structure (see Lemmas 3.6 and 3.8 and Remark 3.7). Note that the weight function here is a little different from Huang-Li-Matsumura [15] due to the discontinuity.

Without loss of generality, we assume the following relation between the viscosity constant $\varepsilon$ and the heat-conducing coefficient $\kappa$ of system (1.1) as in $[20]$ :

$$
\left\{\begin{array}{l}
\kappa=O(\varepsilon), \quad \text { as } \quad \varepsilon \rightarrow 0 \\
\nu \doteq \frac{\kappa(\varepsilon)}{\varepsilon} \geq c>0 \quad \text { for some positive constant } c, \quad \text { as } \quad \varepsilon \rightarrow 0
\end{array}\right.
$$

If $\kappa=\varepsilon=0$ in (1.1), then the corresponding Euler system reads as

$$
\left\{\begin{array}{l}
v_{t}-u_{x}=0, \\
u_{t}+p_{x}=0, \\
\left(e+\frac{u^{2}}{2}\right)_{t}+(p u)_{x}=0 .
\end{array}\right.
$$

It can be easily computed that the eigenvalues of the Jacobi matrix of the flux function to (1.4) are

$$
\lambda_{1}=-\sqrt{\frac{\gamma p}{v}}, \quad \lambda_{2}=0, \quad \lambda_{3}=\sqrt{\frac{\gamma p}{v}} .
$$


It is well known that the first and third characteristic fields of (1.4) are genuinely nonlinear and the second one is linearly degenerate (see [25]).

For the Euler equations, we know that there are three basic wave patterns, shock, rarefaction wave and contact discontinuity. And the Riemann solution to the Euler equations has a basic wave pattern consisting the superposition of these three waves with the contact discontinuity in the middle. For later use, let us firstly recall the wave curves for the two types of basic waves studied in this paper.

Given the right end state $\left(v_{+}, u_{+}, \theta_{+}\right)$with $v_{+}, \theta_{+}>0$, the following wave curves in the phase space $\{(v, u, \theta) \mid v>0, \theta>0\}$ are defined for the Euler equations.

- Contact discontinuity curve:

$$
C D\left(v_{+}, u_{+}, \theta_{+}\right)=\left\{(v, u, \theta) \mid u=u_{+}, p=p_{+}, v \not \equiv v_{+}\right\} .
$$

- $i$-Rarefaction wave curve $(i=1,3)$ :

$R_{i}\left(v_{+}, u_{+}, \theta_{+}\right):=\left\{(v, u, \theta) \mid u<u_{+}, u=u_{+}-\int_{v_{+}}^{v} \lambda_{i}\left(\eta, s_{+}\right) d \eta, s(v, \theta)=s_{+}\right\}$,

where $s_{+}=s\left(v_{+}, \theta_{+}\right)$and $\lambda_{i}=\lambda_{i}(v, s)$ defined in (1.5) is the $i$-th characteristic speed of the Euler system (1.4).

Now, we define the solution profile that consists of the superposition of two rarefaction waves and a contact discontinuity. Let $\left(v_{-}, u_{-}, \theta_{-}\right) \in$ $R_{1}-C D-R_{3}\left(v_{+}, u_{+}, \theta_{+}\right)$. Then, there exist uniquely two intermediate states $\left(v_{*}, u_{*}, \theta_{*}\right)$ and $\left(v^{*}, u^{*}, \theta^{*}\right)$, such that $\left(v_{*}, u_{*}, \theta_{*}\right) \in R_{1}\left(v_{-}, u_{-}, \theta_{-}\right),\left(v_{*}, u_{*}\right.$, $\left.\theta_{*}\right) \in C D\left(v^{*}, u^{*}, \theta^{*}\right)$ and $\left(v^{*}, u^{*}, \theta^{*}\right) \in R_{3}\left(v_{+}, u_{+}, \theta_{+}\right)$.

Thus, the wave pattern $(\bar{V}, \bar{U}, \bar{\Theta})(t, x)$ consisting of 1-rarefaction wave, 2-contact discontinuity and 3-rarefaction wave that solves the corresponding Riemann problem of the Euler system (1.4) can be defined by

$$
\left(\begin{array}{c}
\bar{V} \\
\bar{U} \\
\bar{\Theta}
\end{array}\right)(t, x)=\left(\begin{array}{c}
v^{r_{1}}+v^{c d}+v^{r_{3}} \\
u^{r_{1}}+u^{c d}+u^{r_{3}} \\
\theta^{r_{1}}+\theta^{c d}+\theta^{r_{3}}
\end{array}\right)(t, x)-\left(\begin{array}{c}
v_{*}+v^{*} \\
u_{*}+u^{*} \\
\theta_{*}+\theta^{*}
\end{array}\right)
$$

where $\left(v^{r_{1}}, u^{r_{1}}, \theta^{r_{1}}\right)(t, x)$ is the 1-rarefaction wave defined in (1.7) with the right state $\left(v_{+}, u_{+}, \theta_{+}\right)$replaced by $\left(v_{*}, u_{*}, \theta_{*}\right),\left(v^{c d}, u^{c d}, \theta^{c d}\right)(t, x)$ is the contact discontinuity defined in (1.6) with the states $\left(v_{-}, u_{-}, \theta_{-}\right)$and $\left(v_{+}, u_{+}\right.$, $\left.\theta_{+}\right)$replaced by $\left(v_{*}, u_{*}, \theta_{*}\right)$ and $\left(v^{*}, u^{*}, \theta^{*}\right)$ respectively, and $\left(v^{r_{3}}, u^{r_{3}}, \theta^{r_{3}}\right)(t, x)$ is the 3-rarefaction wave defined in (1.7) with the left state $\left(v_{-}, u_{-}, \theta_{-}\right)$replaced by $\left(v^{*}, u^{*}, \theta^{*}\right)$. 
Now we state the main result as follows.

Theorem 1.1. Given a Riemann solution $(\bar{V}, \bar{U}, \bar{\Theta})(t, x)$ defined in (1.8), which is superposition of two rarefaction waves and a contact discontinuity for the Euler system (1.4), there exist small positive constants $\delta_{0}$ and $\varepsilon_{0}$, such that if $\varepsilon \leq \varepsilon_{0}$ and the wave strength $\delta \doteq\left|\left(v_{+}-v_{-}, u_{+}-u_{-}, \theta_{+}-\theta_{-}\right)\right| \leq \delta_{0}$, then the compressible Navier-Stokes equations (1.1) with (1.2) and (1.3) admits a unique global piece-wise smooth solution $\left(v^{\varepsilon}, u^{\varepsilon}, \theta^{\varepsilon}\right)(t, x)$ satisfying that

- The quantities $u^{\varepsilon}, \theta^{\varepsilon}, p\left(v^{\varepsilon}, \theta^{\varepsilon}\right)-\varepsilon \frac{u_{x}^{\varepsilon}}{v^{\varepsilon}}$ and $\frac{\theta_{x}^{\varepsilon}}{v^{\varepsilon}}$ are continuous for $t>0$, and the jumps in $v^{\varepsilon}, u_{x}^{\varepsilon}, \theta_{x}^{\varepsilon}$ at $x=0$ satisfies

$$
\left|\left(\left[v^{\varepsilon}(t, 0)\right],\left[u_{x}^{\varepsilon}(t, 0)\right],\left[\theta_{x}^{\varepsilon}(t, 0)\right]\right)\right| \leq C e^{-\frac{c t}{\varepsilon}},
$$

where the constants $C$ and $c$ are independent of $t$ and $\varepsilon$.

- Moreover, under the condition (1.3), it holds that

(1.9) $\lim _{\varepsilon \rightarrow 0} \sup _{(t, x) \in \Sigma_{h}}\left|\left(v^{\varepsilon}, u^{\varepsilon}, \theta^{\varepsilon}\right)(t, x)-(\bar{V}, \bar{U}, \bar{\Theta})(t, x)\right|=0, \quad \forall h>0$,

where $\Sigma_{h}=\left\{(t, x) \mid t \geq h, \frac{|x|}{\sqrt{\varepsilon+t}} \geq h \varepsilon^{\alpha}, 0 \leq \alpha<\frac{1}{2}\right\}$.

Remark 1.2. Theorem 1.1 shows that, away from the initial time $t=0$ and the contact discontinuity located at $x=0$, there exists a unique global solution $\left(v^{\varepsilon}, u^{\varepsilon}, \theta^{\varepsilon}\right)(t, x)$ of the compressible Navier-Stokes equations (1.1) which converges to the Riemann solution $(\bar{V}, \bar{U}, \bar{\Theta})(t, x)$ consisting of two rarefaction waves and a contact discontinuity when $\varepsilon$ and $\kappa$ satisfy the relation (1.3) and $\varepsilon$ tends to zero. Moreover, the convergence is uniform on the set $\Sigma_{h}$ for any $h>0$.

Notations. In the paper, we always use the notation $f_{\mathbf{R}}=\int_{\mathbf{R}^{+}}+\int_{\mathbf{R}^{-}},\|\cdot\|$ to denote the usual $L^{2}(\mathbf{R})$ norm, $\| \cdot H$ to denote the piecewise $L^{2}$ norm, that is, $\left\|f H^{2}=f_{\mathbf{R}} f^{2} d y \cdot\right\| \cdot \|_{1}$ and $\| \cdot H_{1}$ represent the $H^{1}(\mathbf{R})$ norm and piece-wise $H^{1}\left(\mathbf{R}^{ \pm}\right)$norm, respectively. And the notation [.] represents the jump of the function - at $x=0$ or $y=0$ if without confusion.

\section{Approximate profiles}

Introduce the following scaled variables

$$
y=\frac{x}{\varepsilon}, \quad \tau=\frac{t}{\varepsilon}
$$


and set

$$
\left(v^{\varepsilon}, u^{\varepsilon}, \theta^{\varepsilon}\right)(t, x)=(v, u, \theta)(\tau, y) .
$$

Then the new unknown functions $(v, u, \theta)(\tau, y)$ satisfies the system

$$
\left\{\begin{array}{l}
v_{\tau}-u_{y}=0, \\
u_{\tau}+p_{y}=\left(\frac{u_{y}}{v}\right)_{y}, \\
\frac{R}{\gamma-1} \theta_{\tau}+p u_{y}=\nu\left(\frac{\theta_{y}}{v}\right)_{y}+\frac{u_{y}^{2}}{v},
\end{array}\right.
$$

with the scaled heat conductivity $\nu=\frac{\kappa}{\varepsilon}$ in (1.3) satisfying

$\nu_{0} \leq \nu \leq \nu_{1}$, uniformly in $\varepsilon$ as $\varepsilon \rightarrow 0+$, for some positive constants $\nu_{0}$ and $\nu_{1}$.

Note that the Riemann solution $(\bar{V}, \bar{U}, \bar{\Theta})(t, x)$ in $(1.8)$ is invariant under the scaling transformation (2.1), thus to prove the limit (1.9) in Theorem 1.1 , it is sufficient to show the following limit

$$
\lim _{\varepsilon \rightarrow 0} \sup _{(\tau, y) \in \Sigma_{h}^{1}}|(v, u, \theta)(\tau, y)-(\bar{V}, \bar{U}, \bar{\Theta})(\tau, y)|=0, \quad \forall h>0,
$$

where $\Sigma_{h}^{1}$ is the corresponding region of $\Sigma_{h}$ in the new coordinates $(\tau, y)$ defined by

$$
\Sigma_{h}^{1}=\left\{(\tau, y) \mid \tau \geq \frac{h}{\varepsilon}, \frac{|y|}{\sqrt{1+\tau}} \geq \frac{h}{\varepsilon^{\frac{1}{2}-\alpha}}, 0 \leq \alpha<\frac{1}{2}\right\} .
$$

Now we study the Navier-Stokes equations (2.2). The corresponding wave profiles to (1.6) and (1.7) can be defined approximately as follows. We start from the viscous contact wave to (1.6).

\subsection{Viscous contact wave}

If $\left(v_{-}, u_{-}, \theta_{-}\right) \in C D\left(v_{+}, u_{+}, \theta_{+}\right)$, i.e.,

$$
u_{-}=u_{+}, p_{-}=p_{+}, v_{-} \neq v_{+},
$$

then the Riemann problem, that is, the Euler system (1.4) with Riemann initial data

$$
(v, u, \theta)(\tau=0, y)= \begin{cases}\left(v_{-}, u_{-}, \theta_{-}\right), & y<0, \\ \left(v_{+}, u_{+}, \theta_{+}\right), & y>0,\end{cases}
$$


admits a single contact discontinuity solution

$$
\left(v^{c d}, u^{c d}, \theta^{c d}\right)(\tau, y)= \begin{cases}\left(v_{-}, u_{+}, \theta_{-}\right), & y<u_{+} \tau, \tau>0 \\ \left(v_{+}, u_{+}, \theta_{+}\right), & y>u_{+} \tau, \tau>0 .\end{cases}
$$

As in [16], the viscous version of the above contact discontinuity, called viscous contact wave $\left(V^{C D}, U^{C D}, \Theta^{C D}\right)(\tau, y)$, can be defined as follows. Since it is expected that

$$
P^{C D} \approx p_{+}=p_{-}, \quad \text { and }\left|U^{C D}-u_{+}\right| \ll 1,
$$

the leading order of the energy equation $(2.2)_{3}$ is

$$
\frac{R}{\gamma-1} \Theta_{\tau}+p_{+} U_{y}=\nu\left(\frac{\Theta_{y}}{V}\right)_{y} .
$$

Then, similar to [15] or [17], one can get the following nonlinear diffusion equation

$$
\Theta_{\tau}=a\left(\frac{\Theta_{y}}{\Theta}\right)_{y}, \quad \Theta(\tau, \pm)=\theta_{ \pm}, \quad a=\frac{\nu p_{+}(\gamma-1)}{R^{2} \gamma} .
$$

The above diffusion equation has a unique self-similar solution $\hat{\Theta}(\tau, y)=$ $\hat{\Theta}\left(\frac{y}{\sqrt{1+\tau}}\right)$.

Thus, the viscous contact wave $\left(V^{C D}, U^{C D}, \Theta^{C D}\right)(\tau, y)$ can be defined by

$$
\begin{aligned}
& V^{C D}(\tau, y)=\frac{R \hat{\Theta}(\tau, y)}{p_{+}}, \\
& U^{C D}(\tau, y)=u_{+}+\frac{\nu(\gamma-1)}{R \gamma} \frac{\hat{\Theta}_{y}(\tau, y)}{\hat{\Theta}(\tau, y)}, \\
& \Theta^{C D}(\tau, y)=\hat{\Theta}(\tau, y)+\frac{R \gamma-\nu(\gamma-1)}{\gamma p_{+}} \hat{\Theta}_{\tau} .
\end{aligned}
$$

Here, it is straightforward to check that the viscous contact wave defined in (2.5) satisfies

$$
\left|\hat{\Theta}-\theta_{ \pm}\right|+(1+\tau)^{\frac{1}{2}}\left|\hat{\Theta}_{y}\right|+(1+\tau)\left|\hat{\Theta}_{y y}\right|=O(1) \delta^{C D} e^{-\frac{c_{0} y^{2}}{1+\tau}}, \quad \text { as }|y| \rightarrow+\infty,
$$

where $\delta^{C D}=\left|\theta_{+}-\theta_{-}\right|$represents the strength of the viscous contact wave and $c_{0}$ is a positive constant. Note that in (2.5), the higher order term 
$\frac{R \gamma-\nu(\gamma-1)}{\gamma p_{+}} \hat{\Theta}_{\tau}$ is introduced in $\Theta^{C D}(\tau, y)$ to make the viscous contact wave $\left(V^{C D}, U^{C D}, \Theta^{C D}\right)(\tau, y)$ satisfy the momentum equation exactly, see also [23, 24]. Correspondingly, $\left(V^{C D}, U^{C D}, \Theta^{C D}\right)(\tau, y)$ satisfies the system

$$
\left\{\begin{array}{l}
V_{\tau}^{C D}-U_{y}^{C D}=0 \\
U_{\tau}^{C D}+P_{y}^{C D}=\left(\frac{U_{y}^{C D}}{V^{C D}}\right)_{y}, \\
\frac{R}{\gamma-1} \Theta_{\tau}^{C D}+P^{C D} U_{y}^{C D}=\nu\left(\frac{\Theta_{y}^{C D}}{V^{C D}}\right)_{y}+\frac{\left(U_{y}^{C D}\right)^{2}}{V^{C D}}+Q^{C D},
\end{array}\right.
$$

where $P^{C D}=\frac{R \Theta^{C D}}{V^{C D}}$ and the error term $Q^{C D}$ satisfies

$$
Q^{C D}=O(1) \delta^{C D}(1+\tau)^{-2} e^{-\frac{c_{0} y^{2}}{1+\tau}}, \quad \text { as }|y| \rightarrow+\infty,
$$

for some positive constant $c_{0}$.

\subsection{Approximate rarefaction waves}

We now turn to the approximate rarefaction waves to (1.7). Since there is no exact rarefaction wave profile for the Navier-Stokes equations, the following approximate rarefaction wave profile, which satisfies the Euler equations, is motivated by [28]. For the completeness of presentation, we include its definition and the properties in this subsection.

If $\left(v_{-}, u_{-}, \theta_{-}\right) \in R_{i}\left(v_{+}, u_{+}, \theta_{+}\right),(i=1,3)$, then there exists an $i$-rarefaction wave $\left(v^{r_{i}}, u^{r_{i}}, \theta^{r_{i}}\right)(y / \tau)$ which is a global solution of the following Riemann problem:

$$
\left\{\begin{array}{l}
v_{\tau}-u_{y}=0, \\
u_{\tau}+p_{y}(v, \theta)=0, \\
\frac{R}{\gamma-1} \theta_{\tau}+p(v, \theta) u_{y}=0, \\
(v, u, \theta)(0, y)= \begin{cases}\left(v_{-}, u_{-}, \theta_{-}\right), & y<0, \\
\left(v_{+}, u_{+}, \theta_{+}\right), & y>0 .\end{cases}
\end{array}\right.
$$

Consider the following inviscid Burgers equation with Riemann data:

$$
\left\{\begin{array}{l}
w_{\tau}+w w_{y}=0, \\
w(\tau=0, y)= \begin{cases}w_{-}, & y<0, \\
w_{+}, & y>0 .\end{cases}
\end{array}\right.
$$


If $w_{-}<w_{+}$, then the Riemann problem (2.10) admits a rarefaction wave solution

$$
w^{r}(\tau, y)=w^{r}\left(\frac{y}{\tau}\right)= \begin{cases}w_{-}, & \frac{y}{\tau} \leq w_{-} \\ \frac{y}{\tau}, & w_{-} \leq \frac{y}{\tau} \leq w_{+} \\ w_{+}, & \frac{y}{\tau} \geq w_{+}\end{cases}
$$

Thus, the Riemann solution in (2.9) can be expressed explicitly through the above rarefaction wave (2.11) to the Burgers equation, that is,

$$
\left\{\begin{array}{l}
s^{r_{i}}(\tau, y)=s\left(v^{r_{i}}(\tau, y), \theta^{r_{i}}(\tau, y)\right)=s_{+} \\
w_{ \pm}=\lambda_{i \pm}:=\lambda_{i}\left(v_{ \pm}, \theta_{ \pm}\right) \\
w^{r}\left(\frac{y}{\tau}\right)=\lambda_{i}\left(v^{r_{i}}(\tau, y), s_{+}\right) \\
u^{r_{i}}(\tau, y)=u_{+}-\int_{v_{+}}^{v^{r_{i}}(\tau, y)} \lambda_{i}\left(v, s_{+}\right) d v
\end{array}\right.
$$

In order to construct the approximate rarefaction wave $\left(V^{R_{i}}, U^{R_{i}}\right.$, $\left.\Theta^{R_{i}}\right)(\tau, y)$ corresponding to $(2.12)$, we first consider the following approximate rarefaction wave to the Burgers equation:

$$
\left\{\begin{array}{l}
w_{\tau}+w w_{y}=0 \\
w(0, y)=w_{0}(y)=\frac{w_{+}+w_{-}}{2}+\frac{w_{+}-w_{-}}{2} \tanh y
\end{array}\right.
$$

Note that the solution $w^{R}(\tau, y)$ of the problem $(2.13)$ is given by

$$
w^{R}(\tau, y)=w_{0}\left(x_{0}(\tau, y)\right), \quad x=x_{0}(\tau, y)+w_{0}\left(x_{0}(\tau, y)\right) \tau
$$

And $w^{R}(\tau, y)$ has the following properties, the proof of which can be found in $[22,28]$ :

Lemma 2.1. Let $w_{-}<w_{+}$, then (2.13) has a unique smooth solution $w^{R}(\tau, y)$ satisfying

(1) $w_{-}<w^{R}(\tau, y)<w_{+},\left(w^{R}\right)_{y}(\tau, y)>0$;

(2) For any $1 \leq p \leq+\infty$, there exists a constant $C$ such that

$$
\begin{aligned}
& \left\|\frac{\partial}{\partial y} w^{R}(\tau, \cdot)\right\|_{L^{p}(\mathbf{R})} \leq C \min \left\{\left(w_{+}-w_{-}\right),\left(w_{+}-w_{-}\right)^{1 / p \tau^{-1+1 / p}}\right\} \\
& \left\|\frac{\partial^{2}}{\partial y^{2}} w^{R}(\tau, \cdot)\right\|_{L^{p}(\mathbf{R})} \leq C \min \left\{\left(w_{+}-w_{-}\right), \tau^{-1}\right\}
\end{aligned}
$$


(3) If $y-w_{-} \tau<0$, then

$$
\begin{aligned}
& \left|w^{R}(\tau, y)-w_{-}\right| \leq\left(w_{+}-w_{-}\right) e^{-2\left|y-w_{-} \tau\right|} \\
& \left|\frac{\partial}{\partial y} w^{R}(\tau, y)\right| \leq 2\left(w_{+}-w_{-}\right) e^{-2\left|y-w_{-} \tau\right|}
\end{aligned}
$$

If $y-w_{+} \tau>0$, then

$$
\begin{aligned}
& \left|w^{R}(\tau, y)-w_{+}\right| \leq\left(w_{+}-w_{-}\right) e^{-2\left|y-w_{+} \tau\right|}, \\
& \left|\frac{\partial}{\partial x} w^{R}(\tau, y)\right| \leq 2\left(w_{+}-w_{-}\right) e^{-2\left|y-w_{+} \tau\right|}
\end{aligned}
$$

(4) $\sup _{y \in \mathbf{R}}\left|w^{R}(\tau, y)-w^{r}\left(\frac{y}{\tau}\right)\right| \leq \min \left\{w_{+}-w_{-}, \frac{1}{\tau} \ln (1+\tau)\right\}$.

Then, corresponding to (2.12), the approximate rarefaction wave profile denoted by $\left(V^{R_{i}}, U^{R_{i}}, \Theta^{R_{i}}\right)(\tau, y)(i=1,3)$ to $(1.7)$ can be defined by

$$
\left\{\begin{array}{l}
S^{R_{i}}(\tau, y)=s\left(V^{R_{i}}(\tau, y), \Theta^{R_{i}}(\tau, y)\right)=s_{+} \\
w_{ \pm}=\lambda_{i \pm}:=\lambda_{i}\left(v_{ \pm}, \theta_{ \pm}\right) \\
w^{R}(1+\tau, y)=\lambda_{i}\left(V^{R_{i}}(\tau, y), s_{+}\right) \\
U^{R_{i}}(\tau, y)=u_{+}-\int_{v_{+}}^{V^{R_{i}}(\tau, y)} \lambda_{i}\left(v, s_{+}\right) d v
\end{array}\right.
$$

Note that $\left(V^{R_{i}}, U^{R_{i}}, \Theta^{R_{i}}\right)(\tau, y)$ defined above satisfies

$$
\left\{\begin{array}{l}
V_{\tau}^{R_{i}}-U_{y}^{R_{i}}=0, \\
U_{\tau}^{R_{i}}+P_{y}^{R_{i}}=0, \\
\frac{R}{\gamma-1} \Theta_{\tau}^{R_{i}}+P^{R_{i}} U_{y}^{R_{i}}=0,
\end{array}\right.
$$

where $P^{R_{i}}=p\left(V^{R_{i}}, \Theta^{R_{i}}\right)$.

By virtue of Lemma 2.1, the properties on the approximate rarefaction waves $\left(V^{R_{i}}, U^{R_{i}}, \Theta^{R_{i}}\right)(\tau, y)$ can be summarized as follows.

Lemma 2.2. The approximate rarefaction waves $\left(V^{R_{i}}, U^{R_{i}}, \Theta^{R_{i}}\right)(\tau, y)(i=$ $1,3)$ constructed in (2.14) have the following properties:

(1) $U_{x}^{R_{i}}(\tau, y)>0$ for $y \in \mathbf{R}, \tau>0$;

(2) For any $1 \leq p \leq+\infty$, the following estimates holds,

$$
\begin{aligned}
& \left\|\left(V^{R_{i}}, U^{R_{i}}, \Theta^{R_{i}}\right)_{y}\right\|_{L^{p}(d y)} \leq C \min \left\{\delta^{R_{i}},\left(\delta^{R_{i}}\right)^{1 / p}(1+\tau)^{-1+1 / p}\right\}, \\
& \left\|\left(V^{R_{i}}, U^{R_{i}}, \Theta^{R_{i}}\right)_{y y}\right\|_{L^{p}(d y)} \leq C \min \left\{\delta^{R_{i}},(1+\tau)^{-1}\right\},
\end{aligned}
$$


where $\delta^{R_{i}}=\left|\left(v_{+}-v_{-}, u_{+}-u_{-}, \theta_{+}-\theta_{-}\right)\right|$is the i-rarefaction wave strength and the positive constant $C$ is independent of $\tau$, but may depend on $p$ and the wave strength;

(3) If $y \geq \lambda_{1+}(1+\tau)$, then

$$
\begin{aligned}
& \left|\left(V^{R_{1}}, U^{R_{1}}, \Theta^{R_{1}}\right)(\tau, y)-\left(v_{-}, u_{-}, \theta_{-}\right)\right| \leq C \delta^{R_{1}} e^{-2\left|y-\lambda_{1+}(1+\tau)\right|}, \\
& \left|\left(V^{R_{1}}, U^{R_{1}}, \Theta^{R_{1}}\right)_{y}(\tau, y)\right| \leq C \delta^{R_{1}} e^{-2\left|y-\lambda_{1+}(1+\tau)\right|} ;
\end{aligned}
$$

If $y \leq \lambda_{3-}(1+\tau)$, then

$$
\begin{aligned}
& \left|\left(V^{R_{3}}, U^{R_{3}}, \Theta^{R_{3}}\right)(\tau, y)-\left(v_{+}, u_{+}, \theta_{+}\right)\right| \leq C \delta^{R_{3}} e^{-2\left|y-\lambda_{3-}(1+\tau)\right|}, \\
& \left|\left(V^{R_{3}}, U^{R_{3}}, \Theta^{R_{3}}\right)_{y}(\tau, y)\right| \leq C \delta^{R_{3}} e^{-2\left|y-\lambda_{3-}(1+\tau)\right|} ;
\end{aligned}
$$

(4) There exists a positive constant $C$, such that for all $\tau>0$,

$$
\sup _{y \in \mathbf{R}}\left|\left(V^{R_{i}}, U^{R_{i}}, \Theta^{R_{i}}\right)(\tau, y)-\left(v^{r_{i}}, u^{r_{i}}, \theta^{r_{i}}\right)\left(\frac{y}{\tau}\right)\right| \leq \frac{C}{1+\tau} \ln (1+\tau) .
$$

\subsection{Superposition of rarefaction waves and contact discontinuity}

Corresponding to (1.8), the approximate wave pattern $(V, U, \Theta)(\tau, y)$ of the compressible Navier-Stokes equations (2.2) can be defined by

$$
\left(\begin{array}{c}
V \\
U \\
\Theta
\end{array}\right)(\tau, y)=\left(\begin{array}{c}
V^{R_{1}}+V^{C D}+V^{R_{3}} \\
U^{R_{1}}+U^{C D}+U^{R_{3}} \\
\Theta^{R_{1}}+\Theta^{C D}+\Theta^{R_{3}}
\end{array}\right)(\tau, y)-\left(\begin{array}{c}
v_{*}+v^{*} \\
u_{*}+u^{*} \\
\theta_{*}+\theta^{*}
\end{array}\right)
$$

where $\left(V^{R_{1}}, U^{R_{1}}, \Theta^{R_{1}}\right)(\tau, y)$ is the approximate 1-rarefaction wave defined in (2.14) with the right state $\left(v_{+}, u_{+}, \theta_{+}\right)$replaced by $\left(v_{*}, u_{*}, \theta_{*}\right),\left(V^{C D}, U^{C D}\right.$, $\left.\Theta^{C D}\right)(\tau, y)$ is the viscous contact wave defined in (2.5) with the states $\left(v_{-}, u_{-}, \theta_{-}\right)$and $\left(v_{+}, u_{+}, \theta_{+}\right)$replaced by $\left(v_{*}, u_{*}, \theta_{*}\right)$ and $\left(v^{*}, u^{*}, \theta^{*}\right)$ respectively, and $\left(V^{R_{3}}, U^{R_{3}}, \Theta^{R_{3}}\right)(\tau, y)$ is the approximate 3 -rarefaction wave defined in (2.14) with the left state $\left(v_{-}, u_{-}, \theta_{-}\right)$replaced by $\left(v^{*}, u^{*}, \theta^{*}\right)$.

Thus, from the properties of the viscous contact wave in (2.6) and the approximate rarefaction wave in Lemma 2.3 , we have the following relation between the approximate wave pattern $(V, U, \Theta)(\tau, y)$ and the exact inviscid wave pattern $(\bar{V}, \bar{U}, \bar{\Theta})(\tau, y)$ of the Euler equations

$$
|(V, U, \Theta)(\tau, y)-(\bar{V}, \bar{U}, \bar{\Theta})(\tau, y)| \leq \frac{C}{1+\tau} \ln (1+\tau)+C \delta^{C D} e^{-\frac{c y^{2}}{1+\tau}}
$$


Hence, to prove the zero dissipation limit (2.3) on the set $\Sigma_{h}^{1}$ defined in (2.4), it is sufficient to show the following time-asymptotic behavior of the solution to (2.2) around the approximate wave profile (2.16), i.e.,

$$
\lim _{\tau \rightarrow+\infty}\|(v, u, \theta)(\tau, \cdot)-(V, U, \Theta)(\tau, \cdot)\|_{L^{\infty}}=0 .
$$

First, by (2.7) and (2.15), the superposition wave profile $(V, U, \Theta)(\tau, y)$ defined in (2.16) satisfies the following system

$$
\left\{\begin{array}{l}
V_{\tau}-U_{y}=0, \\
U_{\tau}+P_{y}=\left(\frac{U_{y}}{V}\right)_{y}+Q_{1}, \\
\frac{R}{\gamma-1} \Theta_{\tau}+P U_{y}=\nu\left(\frac{\Theta_{y}}{V}\right)_{y}+\frac{U_{y}^{2}}{V}+Q_{2},
\end{array}\right.
$$

where $P=p(V, \Theta)$ and

$$
\begin{aligned}
Q_{1} & =\left(P-P^{R_{1}}-P^{C D}-P^{R_{3}}\right)_{y}-\left(\frac{U_{y}}{V}-\frac{U_{y}^{C D}}{V^{C D}}\right)_{y}, \\
Q_{2} & =\left(P U_{y}-P^{R_{1}} U_{y}^{R_{1}}-P^{C D} U_{y}^{C D}-P^{R_{3}} U_{y}^{R_{3}}\right)-\nu\left(\frac{\Theta_{y}}{V}-\frac{\Theta_{y}^{C D}}{V^{C D}}\right)_{y} \\
& -\left(\frac{U_{y}^{2}}{V}-\frac{\left(U_{y}^{C D}\right)^{2}}{V^{C D}}\right)-Q^{C D} .
\end{aligned}
$$

A direct calculation shows that

$$
\begin{aligned}
Q_{1}= & O(1)\left\{\left|\left(V_{y}^{R_{1}}, \Theta_{y}^{R_{1}}\right)\right|\left|\left(V^{C D}-v_{*}, \Theta^{C D}-\theta_{*}, V^{R_{3}}-v^{*}, \Theta^{R_{3}}-\theta^{*}\right)\right|\right. \\
& +\left|\left(V_{y}^{R_{3}}, \Theta_{y}^{R_{3}}\right)\right|\left|\left(V^{R_{1}}-v_{*}, \Theta^{R_{1}}-\theta_{*}, V^{C D}-v^{*}, \Theta^{C D}-\theta^{*}\right)\right| \\
& +\left|\left(V_{y}^{C D}, \Theta_{y}^{C D}, U_{y}^{C D}\right)\right|\left|\left(V^{R_{1}}-v_{*}, \Theta^{R_{1}}-\theta_{*}, V^{R_{3}}-v^{*}, \Theta^{R_{3}}-\theta^{*}\right)\right| \\
& \left.+\left|\left(U_{y}^{C D}, V_{y}^{C D}\right)\right|\left|\left(U_{y}^{R_{1}}, V_{y}^{R_{1}}, U_{y}^{R_{3}}, V_{y}^{R_{3}}\right)\right|+\left|\left(U_{y}^{R_{1}}, V_{y}^{R_{1}}\right)\right|\left|\left(U_{y}^{R_{3}}, V_{y}^{R_{3}}\right)\right|\right\} \\
& +O(1)\left\{\left|U_{y y}^{R_{1}}\right|+\left|U_{y y}^{R_{3}}\right|+\left|U_{y}^{R_{1}}\right|\left|V_{y}^{R_{1}}\right|+\left|U_{y}^{R_{3}}\right|\left|V_{y}^{R_{3}}\right|\right\} \\
:= & Q_{11}+Q_{12} .
\end{aligned}
$$

Similarly, it holds that

$$
Q_{2}=O(1)\left\{\left|U_{y}^{R_{1}}\right|\left|\left(V^{C D}-v_{*}, \Theta^{C D}-\theta_{*}, V^{R_{3}}-v^{*}, \Theta^{R_{3}}-\theta^{*}\right)\right|\right.
$$




$$
\begin{aligned}
& +\left|U_{y}^{R_{3}}\right|\left|\left(V^{R_{1}}-v_{*}, \Theta^{R_{1}}-\theta_{*}, V^{C D}-v^{*}, \Theta^{C D}-\theta^{*}\right)\right| \\
& +\left|\left(U_{y}^{C D}, V_{y}^{C D}, \Theta_{y}^{C D}\right)\right|\left|\left(V^{R_{1}}-v_{*}, \Theta^{R_{1}}-\theta_{*}, V^{R_{3}}-v^{*}, \Theta^{R_{3}}-\theta^{*}\right)\right| \\
& +\left|\left(U_{y}^{C D}, V_{y}^{C D}, \Theta_{y}^{C D}\right)\right|\left|\left(U_{y}^{R_{1}}, V_{y}^{R_{1}}, \Theta_{y}^{R_{1}}, U_{y}^{R_{3}}, V_{y}^{R_{3}}, \Theta_{y}^{R_{1}}\right)\right| \\
& \left.+\left|\left(U_{y}^{R_{1}}, V_{y}^{R_{1}}, \Theta_{y}^{R_{1}}\right)\right|\left|\left(U_{y}^{R_{3}}, V_{y}^{R_{3}}, \Theta_{y}^{R_{3}}\right)\right|\right\} \\
& +O(1)\left\{\left|\Theta_{y y}^{R_{1}}\right|+\left|\Theta_{y y}^{R_{3}}\right|+\left|\left(U_{y}^{R_{1}}, V_{y}^{R_{1}}, \Theta_{y}^{R_{1}}, U_{y}^{R_{3}}, V_{y}^{R_{3}}, \Theta_{y}^{R_{3}}\right)\right|^{2}\right\}+\left|Q^{C D}\right| \\
& :=Q_{21}+Q_{22}+\left|Q^{C D}\right| .
\end{aligned}
$$

Here $Q_{11}$ and $Q_{21}$ represent the wave interaction terms coming from the wave patterns in the different family, $Q_{12}$ and $Q_{22}$ stand for the error terms due to the inviscid approximate rarefaction wave profiles, and $Q^{C D}$ is the error term defined in (2.8) due to the viscous contact wave.

In fact, one can estimate the interaction terms $Q_{11}$ and $Q_{21}$ by dividing the whole domain $\Omega=\{(\tau, y) \mid(\tau, y) \in \mathbf{R} \times \mathbf{R}\}$ into three regions:

$$
\begin{aligned}
& \Omega_{-}=\left\{(\tau, y) \mid 2 y \leq \lambda_{1 *}(1+\tau)\right\}, \\
& \Omega_{C D}=\left\{(\tau, y) \mid \lambda_{1 *}(1+\tau)<2 y<\lambda_{3}^{*}(1+\tau)\right\}, \\
& \Omega_{+}=\left\{(\tau, y) \mid 2 y \geq \lambda_{3}^{*}(1+\tau)\right\},
\end{aligned}
$$

where $\lambda_{1 *}=\lambda_{1}\left(v_{*}, \theta_{*}\right)$ and $\lambda_{3}^{*}=\lambda_{3}\left(v^{*}, \theta^{*}\right)$. Then, in each section the following estimates follow from (2.6) and Lemma 2.2.

- In $\Omega_{-}$,

$$
\begin{aligned}
& \left|\left(V^{R_{3}}-v^{*}, V_{y}^{R_{3}}\right)\right|=O(1) \delta^{R_{3}} e^{-2\left\{|y|+\left|\lambda_{3}^{*}\right|(1+\tau)\right\}} \\
& \begin{aligned}
\left|\left(V^{C D}-v_{*}, V^{C D}-v^{*}, V_{y}^{C D}\right)\right| & =O(1) \delta^{C D} e^{-\frac{C\left\{\left|\lambda_{1 *}\right|(1+\tau)\right\}^{2}}{1+\tau}} \\
& =O(1) \delta^{C D} e^{-C(1+\tau)}
\end{aligned}
\end{aligned}
$$

- In $\Omega_{C D}$,

$$
\begin{aligned}
& \left|\left(V^{R_{1}}-v_{*}, V_{y}^{R_{1}}\right)\right|=O(1) \delta^{R_{1}} e^{-2\left\{|y|+\left|\lambda_{1_{*}}\right|(1+\tau)\right\}}, \\
& \left|\left(V^{R_{3}}-v^{*}, V_{y}^{R_{3}}\right)\right|=O(1) \delta^{R_{3}} e^{-2\left\{|y|+\left|\lambda_{3}^{*}\right|(1+\tau)\right\}}
\end{aligned}
$$

- In $\Omega_{+}$,

$$
\begin{aligned}
\left|\left(V^{R_{1}}-v_{*}, V_{y}^{R_{1}}\right)\right|=O(1) \delta^{R_{1}} e^{-2\left\{|y|+\left|\lambda_{1 *}\right|(1+\tau)\right\}} & \begin{aligned}
\left|\left(V^{C D}-v_{*}, V^{C D}-v^{*}, V_{y}^{C D}\right)\right| & =O(1) \delta^{C D} e^{-\frac{C\left\{\left|\lambda_{3}^{*}\right|(1+\tau)\right\}^{2}}{1+\tau}} \\
& =O(1) \delta^{C D} e^{-C(1+\tau)} .
\end{aligned}
\end{aligned}
$$


Keep in mind that each individual wave strength is controlled by the total wave strength by (1.6) and (1.7), that is,

$$
\delta^{R_{1}}+\delta^{R_{3}}+\delta^{C D} \leq C \delta .
$$

Hence, in summary, it follows from (2.19), (2.20) and the above arguments that

$$
\left|\left(Q_{11}, Q_{21}\right)\right|=O(1) \delta e^{-C\{|y|+(1+\tau)\}},
$$

for some positive constant $C$ independent of $\tau$ and $y$.

\section{Proof of the main result}

In this section, we shall prove the main result Theorem 1.1. By virtue of the arguments in Section 2.3, it is sufficient to show (2.18) besides the regularity of the solution. To this end, we first reformulate the problem.

\subsection{Reformulation of the problem}

Set the perturbation around the wave profile $(V, U, \Theta)(\tau, y)$ by

$$
(\phi, \psi, \zeta)(\tau, y)=(v, u, \theta)(\tau, y)-(V, U, \Theta)(\tau, y) .
$$

Then, after a straightforward calculation, the perturbation $(\phi, \psi, \zeta)(\tau, y)$ satisfies the system

$$
\left\{\begin{array}{l}
\phi_{\tau}-\psi_{y}=0 \\
\psi_{\tau}+(p-P)_{y}=\left(\frac{u_{y}}{v}-\frac{U_{y}}{V}\right)_{y}-Q_{1} \\
\frac{R}{\gamma-1} \zeta_{\tau}+\left(p u_{y}-P U_{y}\right)=\nu\left(\frac{\theta_{y}}{v}-\frac{\Theta_{y}}{V}\right)_{y}+\left(\frac{u_{y}^{2}}{v}-\frac{U_{y}^{2}}{V}\right)-Q_{2} \\
(\phi, \psi, \zeta)(\tau=0, y)=\left(\phi_{0}, \psi_{0}, \zeta_{0}\right)(y)
\end{array}\right.
$$

where the initial data $\left(\phi_{0}, \psi_{0}, \zeta_{0}\right)(y)$ and its derivatives are sufficiently smooth away from but up to $y=0$, and

$$
\left(\phi_{0}, \psi_{0}, \zeta_{0}\right)(y) \in L^{2}(\mathbf{R}), \phi_{0 y} \in L^{2}\left(\mathbf{R}^{ \pm}\right) .
$$

For simplicity, denote

$$
\mathcal{N}_{0}:=\left\|\left(\phi_{0}, \psi_{0}, \zeta_{0}\right)\right\|^{2}+\| \phi_{0 y} \#^{2} .
$$

In order to prove (2.18), we easily see that it suffices to show 
Proposition 3.1. There exists a positive constant $\delta_{0}$, such that if the wave strength $\delta$ and the initial data satisfy

$$
\delta+\mathcal{N}_{0} \leq \delta_{0}
$$

then the problem (3.1) admits a unique global solution $(\phi, \psi, \zeta)(t, y)$ satisfying

(i) There exists a positive constant $C$ independent of $t$, such that

$$
\begin{aligned}
& \sup _{\tau \geq 0}\left(\|(\phi, \psi, \zeta)(\tau, \cdot)\|^{2}+\left\|\phi_{y}(\tau, \cdot)\right\|^{2}\right)+\int_{0}^{+\infty} \|\left(\phi_{y}, \psi_{y}, \zeta_{y}\right)(\tau, \cdot) \#^{2} d \tau \\
& \quad \leq C\left(\mathcal{N}_{0}+\delta^{\frac{1}{4}}\right) .
\end{aligned}
$$

(ii) For any $\tau_{0}>0$, there exists a positive constant $C=C\left(\tau_{0}\right)$, such that

$$
\begin{aligned}
& \sup _{\tau \geq \tau_{0}}\left\|\left(\psi_{y}, \zeta_{y}, \psi_{\tau}, \zeta_{\tau}\right)(\tau, \cdot) \#^{2}+\int_{\tau_{0}}^{+\infty}\right\|\left(\psi_{y y}, \zeta_{y y}, \psi_{y \tau}, \zeta_{y \tau}\right)(\tau, \cdot) \|^{2} d \tau \\
& \leq C\left(\tau_{0}\right)\left(\mathcal{N}_{0}+\delta^{\frac{1}{4}}\right) .
\end{aligned}
$$

(iii) The jump condition of $\phi(\tau, y)$ at $y=0$ admits the bound

$$
|[\phi](\tau)| \leq C e^{-c \tau}
$$

where the positive constants $C$ and $c$ are independent of $\tau \in(0,+\infty)$.

Assume that Proposition 3.1 holds, then for any $\tau_{0}>0$, one has

$$
\int_{\tau_{0}}^{+\infty}\left(\left\|\left(\phi_{y}, \psi_{y}, \zeta_{y}\right)\right\|^{2}+\left|\frac{d}{d \tau}\left\|\left(\phi_{y}, \psi_{y}, \zeta_{y}\right)\right\|^{2}\right|\right) d \tau<+\infty
$$

whence,

$$
\lim _{\tau \rightarrow \infty} \|\left(\phi_{y}, \psi_{y}, \zeta_{y}\right) \mathbb{H}^{2}=0,
$$

which, together with Proposition 3.1 and Sobolev's inequality, implies that

$$
\begin{aligned}
\lim _{\tau \rightarrow \infty} \sup _{y \neq 0}\|(\phi, \psi, \zeta)\|_{L^{\infty}}^{2} & \leq C \lim _{\tau \rightarrow \infty}\|(\phi, \psi, \zeta)\| \|\left(\phi_{y}, \psi_{y}, \zeta_{y}\right) \# \\
& \leq C \lim _{\tau \rightarrow \infty} \|\left(\phi_{y}, \psi_{y}, \zeta_{y}\right) \#=0 .
\end{aligned}
$$

The above inequality combined with (3.2) gives (2.18). Thus, the main result Theorem 1.1 follows from (2.18) and (2.17). 
Denote

$$
\begin{aligned}
N\left(\tau_{*}, \tau^{*}\right)= & \sup _{\tau \in\left[\tau_{*}, \tau^{*}\right]}\left\{\|(\phi, \psi, \zeta)(\tau, \cdot)\|^{2}+\|\left(\phi_{y}, \psi_{y}, \zeta_{y}\right)(\tau, \cdot) H^{2}\right. \\
& \left.+\left\|\left(\psi_{\tau}, \zeta_{\tau}\right)(\tau, \cdot)\right\|^{2}\right\}, \\
N\left(\tau_{*}\right)= & N\left(\tau_{*}, \tau_{*}\right),
\end{aligned}
$$

and define the solution space by

$$
X\left[\tau_{*}, \tau^{*}\right]=\left\{\begin{array}{ll}
(\phi, \psi, \zeta) & \begin{array}{l}
(\phi, \psi, \zeta)(\tau, y) \in C\left(\left[\tau_{*}, \tau^{*}\right] ; H^{1}\left(\mathbf{R}^{ \pm}\right)\right), \\
\left(\psi_{y}, \zeta_{y}\right) \in L^{2}\left(\tau_{*}, \tau^{*} ; H^{1}\left(\mathbf{R}^{ \pm}\right)\right), \\
\phi_{y} \in L^{2}\left(\tau_{*}, \tau^{*} ; L^{2}\left(\mathbf{R}^{ \pm}\right)\right), \\
\left(\psi_{\tau}, \zeta_{\tau}\right) \in L^{\infty}\left(\tau_{*}, \tau^{*} ; L^{2}\left(\mathbf{R}^{ \pm}\right)\right) \cap L^{2}\left(\tau_{*}, \tau^{*} ; H^{1}\left(\mathbf{R}^{ \pm}\right)\right) .
\end{array}
\end{array}\right\}
$$

Since the local existence of solutions to (3.1) is proved in [9], we just state it and omit its proof for brevity.

Proposition 3.2. (Local existence) Suppose that $\mathcal{N}_{0}$ and the wave strength $\delta$ are suitably small such that inf $v_{0}$ and inf $\theta_{0}$ are positive. Then there exists a positive time $\tau_{0}=\tau_{0}(N(0), \delta)>0$, such that the Cauchy problem (3.1) admits a unique solution $(\phi, \psi, \zeta)(\tau, y) \in X\left[0, \tau_{0}\right]$ satisfying

$$
A\left(\tau_{0}\right)+B\left(\tau_{0}\right)+F\left(\tau_{0}\right) \leq C\left(\mathcal{N}_{0}+\delta\right),
$$

where

$$
\begin{aligned}
& A\left(\tau_{0}\right)=\sup _{0 \leq \tau \leq \tau_{0}}\left\{\|(\phi, \psi, \zeta)(\tau, \cdot)\|^{2}+\left\|\phi_{y}\right\|^{2}\right\}+\int_{0}^{\tau_{0}}\left\|\left(\psi_{y}, \zeta_{y}\right)\right\|^{2} d \tau, \\
& B\left(\tau_{0}\right)=\sup _{0 \leq \tau \leq \tau_{0}}\left\{g(\tau)^{\frac{1}{2}}\left\|\psi_{y}\right\|^{2}+g(\tau) \| \phi_{y} \#^{2}\right\} \\
& \quad+\int_{0}^{\tau_{0}} g(\tau)^{\frac{1}{2}+\vartheta}\left(\left\|\psi_{\tau}\right\|^{2}+\left\|\left(\frac{u_{y}}{v}\right)_{y}\right\|^{2}\right) d \tau \\
& \quad+\int_{0}^{\tau_{0}} g(\tau)\left(\left\|\psi_{y}^{2}\right\|^{2}+\left\|\theta_{\tau}\right\|^{2}+\|\left(\frac{\theta_{y}}{v}\right)_{y} H^{2}\right) d \tau, \\
& F\left(\tau_{0}\right)=\sup _{0 \leq \tau \leq \tau_{0}}\left\{g(\tau)^{\frac{3}{2}+\vartheta}\left(\left\|\psi_{\tau}\right\|^{2}+\|\left(\frac{u_{y}}{v}\right)_{y} \#^{2}\right)+g(\tau)^{3}\left(\left\|\zeta_{\tau}\right\|^{2}+\|\left(\frac{\theta_{y}}{v}\right)_{y} H^{2}\right)\right\} \\
& \left.\quad+\int_{0}^{\tau_{0}} g(\tau)^{\frac{3}{2}+\vartheta}\left\|\psi_{y \tau}\right\|^{2}+g(\tau)^{3}\left\|\zeta_{y \tau}\right\|^{2}\right) d \tau,
\end{aligned}
$$

with $g(\tau)=\tau \wedge 1=\min \{\tau, 1\}$ and $\vartheta \in(0,1)$. Moreover, $v, u, \theta$ have the same regularity as in Theorem 1.1. Thus, $v, u_{x}, \theta_{x}$ have one-side limit at $y=0$ 
and satisfy the jump conditions

$$
\left[p-\frac{u_{y}}{v}\right]=\left[\frac{\theta_{y}}{v}\right]=0 .
$$

Finally, one has the following estimate on the jump at $y=0$,

$$
|[v](\tau)| \leq C \delta e^{-c \tau}, \quad \tau>0
$$

for some positive constants $C$ and $c$ independent of $\tau$.

Hence, in view of the local existence Proposition 3.2 and the standard continuation process, we see that to prove Proposition 3.1, it suffices to show the following (uniform) a priori estimate.

Proposition 3.3. (A priori estimate) Suppose that the Cauchy problem (3.1) has a solution $(\phi, \psi, \zeta)(\tau, y) \in X\left[\tau_{1}, \tau_{2}\right]$. There exists a positive constant $\eta_{1}$, such that if

$$
N\left(\tau_{1}, \tau_{2}\right)+\delta \leq \eta_{1}
$$

then,

$$
\begin{aligned}
& N\left(\tau_{1}, \tau_{2}\right)+\int_{\tau_{1}}^{\tau_{2}}\left\{\left\|\phi_{y}(\tau, \cdot)\right\|^{2}+\left\|\left(\psi_{y}, \zeta_{y}\right)(\tau, \cdot) H_{1}^{2}+\right\|\left(\psi_{y \tau}, \zeta_{y \tau}\right) \|^{2}(\tau, \cdot)\right\} d \tau \\
& \quad \leq C\left(N\left(\tau_{1}\right)+\delta^{\frac{1}{4}}\right),
\end{aligned}
$$

where the positive constant $C$ is independent of $\tau$.

\subsection{Energy estimates}

In this section we will derive the a priori estimate given in Proposition 3.3. Note that under the a priori assumption (3.3), if $\eta \ll 1$, then it holds that

$$
\inf _{\left[\tau_{1}, \tau_{2}\right] \times \mathbf{R}}\{(V+\phi, \Theta+\zeta)(\tau, y)\} \geq C_{0}
$$

for some positive constant $C_{0}$. First, one has the following Lemma:

Lemma 3.4. Under the assumptions of Proposition 3.3, there exists a constant $C>0$, such that for any $\tau \in\left[\tau_{1}, \tau_{2}\right]$,

$$
\left\|\left(\phi, \psi, \zeta, \phi_{y}\right)(\tau, \cdot)\right\|^{2}+\int_{\tau_{1}}^{\tau}\left\{\left\|\sqrt{\left(U_{y}^{R_{1}}, U_{y}^{R_{3}}\right)}(\phi, \zeta)\right\|^{2}+\left\|\left(\phi_{y}, \psi_{y}, \zeta_{y}\right)\right\|^{2}\right\} d \tau
$$




$$
\begin{aligned}
\leq & C\left\|\left(\phi, \psi, \zeta, \phi_{y}\right) H^{2}\left(\tau_{1}\right)+C \int_{\tau_{1}}^{\tau}(1+\tau)^{-\frac{7}{6}}\right\|(\phi, \psi, \zeta)(\cdot, \tau) \|^{2} d \tau+C \delta^{\frac{1}{4}} \\
& +C \delta \int_{\tau_{1}}^{\tau} f_{\mathbf{R}}(1+\tau)^{-1} e^{-\frac{c_{0} y^{2}}{1+\tau}}|(\phi, \zeta)|^{2} d y d \tau .
\end{aligned}
$$

Proof: Let

$$
\Phi(z)=z-1-\ln z .
$$

Arguing similarly to that in [15] or [17], one can get the following equality (3.5)

$$
\begin{aligned}
& I_{1 \tau}(\tau, y)+H_{1 y}(\tau, y)+\frac{\Theta \psi_{y}^{2}}{v \theta}+\nu \frac{\Theta \zeta_{y}^{2}}{v \theta^{2}}+P\left(U_{y}^{R_{1}}+U_{y}^{R_{3}}\right)\left(\Phi\left(\frac{\theta V}{v \Theta}\right)+\gamma \Phi\left(\frac{v}{V}\right)\right) \\
& =Q_{3}-Q_{1} \psi-Q_{2} \frac{\zeta}{\theta},
\end{aligned}
$$

where

$$
\begin{aligned}
I_{1}(\tau, y) & =R \Theta \Phi\left(\frac{v}{V}\right)+\frac{\psi^{2}}{2}+\frac{R \Theta}{\gamma-1} \Phi\left(\frac{\theta}{\Theta}\right), \\
H_{1}(\tau, y) & =(p-P) \psi-\left(\frac{u_{y}}{v}-\frac{U_{y}}{V}\right) \psi-\nu\left(\frac{\theta_{y}}{v}-\frac{\Theta_{y}}{V}\right) \frac{\zeta}{\theta},
\end{aligned}
$$

and

$$
\begin{aligned}
Q_{3}= & -P U_{y}^{C D}\left(\Phi\left(\frac{\theta V}{v \Theta}\right)+\gamma \Phi\left(\frac{v}{V}\right)\right)+\left(\nu\left(\frac{\Theta_{y}}{V}\right)_{y}+\frac{U_{y}^{2}}{V}+Q_{2}\right)\left\{(\gamma-1) \Phi\left(\frac{v}{V}\right)\right. \\
& \left.+\Phi\left(\frac{\theta}{\Theta}\right)-\frac{\zeta^{2}}{\theta \Theta}\right\}-\left(\frac{1}{v}-\frac{1}{V}\right) U_{y} \psi_{y}+\left(\frac{1}{v}-\frac{1}{V}\right) U_{y}^{2} \frac{\zeta}{\theta}+2 \frac{\zeta \psi_{y} U_{y}}{v \theta}+\nu \frac{\Theta_{y} \zeta_{y} \zeta}{v \theta^{2}} \\
& -\nu\left(\frac{1}{v}-\frac{1}{V}\right) \frac{\Theta \Theta_{y} \zeta_{y}}{\theta^{2}}+\nu\left(\frac{1}{v}-\frac{1}{V}\right) \frac{\zeta \Theta_{y}^{2}}{\theta^{2}} .
\end{aligned}
$$

Integration of the equality (3.5) with respect to $y$ and $\tau$ over $\mathbf{R}^{ \pm} \times\left[\tau_{1}, \tau\right]$ yields that

$$
\begin{gathered}
\int I_{1}(\tau, y) d y+\int_{\tau_{1}}^{\tau}\left[H_{1}\right](\tau) d \tau+\int_{\tau_{1}}^{\tau} f_{\mathbf{R}}\left(\frac{\Theta \psi_{y}^{2}}{v \theta}+\nu \frac{\Theta \zeta_{y}^{2}}{v \theta^{2}}\right) d y d \tau \\
\quad+\int_{\tau_{1}}^{\tau} f_{\mathbf{R}} P\left(U_{y}^{R_{1}}+U_{y}^{R_{3}}\right)\left(\Phi\left(\frac{\theta V}{v \Theta}\right)+\gamma \Phi\left(\frac{v}{V}\right)\right) d y d \tau \\
=\int I_{1}\left(\tau_{1}, y\right) d y+\int_{\tau_{1}}^{\tau} f_{\mathbf{R}}\left(Q_{3}-Q_{1} \psi-Q_{2} \frac{\zeta}{\theta}\right) d y d \tau
\end{gathered}
$$


It is easy to observe that the jump of $H_{1}$ in (3.6) across $y=0$ vanishes, i.e.,

$$
\begin{aligned}
{\left[H_{1}\right](\tau) } & =\left[\left(p-\frac{u_{y}}{v}\right) \psi\right]-\left[\left(P-\frac{U_{y}}{V}\right) \psi\right]-\nu\left[\left(\frac{\theta_{y}}{v}-\frac{\Theta_{y}}{V}\right) \frac{\zeta}{\theta}\right] \\
& =\left[p-\frac{u_{y}}{v}\right] \psi(\tau, 0)-\left[P-\frac{U_{y}}{V}\right] \psi(\tau, 0)-\nu\left(\left[\frac{\theta_{y}}{v}\right]-\left[\frac{\Theta_{y}}{V}\right]\right) \frac{\zeta(\tau, 0)}{\theta(\tau, 0)}=0 .
\end{aligned}
$$

Recalling that

$$
\Phi(1)=\Phi^{\prime}(1)=0, \quad \Phi^{\prime \prime}(z)=z^{-2}>0
$$

there exists a positive constant $C$, such that if $z$ is near 1 , then

$$
C^{-1}(z-1)^{2} \leq \Phi(z) \leq C(z-1)^{2} .
$$

Thus under the a priori assumptions (3.3), one gets

$$
C^{-1}|\phi|^{2} \leq \Phi\left(\frac{v}{V}\right) \leq C|\phi|^{2}, \quad C^{-1}|\zeta|^{2} \leq \Phi\left(\frac{\theta}{\Theta}\right) \leq C|\zeta|^{2}
$$

and

$$
C^{-1}|(\phi, \zeta)|^{2} \leq \Phi\left(\frac{\theta V}{v \Theta}\right)+\gamma \Phi\left(\frac{v}{V}\right) \leq C|(\phi, \zeta)|^{2}
$$

Now it follows from (3.7), (3.9), (3.10) and Cauchy-Schwarz's inequality that (3.11)

$$
\begin{aligned}
\left|Q_{3}\right| \leq & \frac{\Theta \psi_{y}^{2}}{4 v \theta}+\frac{\nu \Theta \zeta_{y}^{2}}{4 v \theta^{2}}+C\left\{\left(\left|\Theta_{y}^{C D}\right|^{2},\left|\Theta_{y y}^{C D}\right|\right)+\left(\left|\left(V_{y}^{R_{1}}, U_{y}^{R_{1}}, \Theta_{y}^{R_{1}}\right)\right|^{2},\left|\Theta_{y y}^{R_{1}}\right|\right)\right. \\
& \left.+\left(\left|\left(V_{y}^{R_{3}}, U_{y}^{R_{3}}, \Theta_{y}^{R_{3}}\right)\right|^{2},\left|\Theta_{y y}^{R_{3}}\right|\right)+\left|Q_{2}\right|\right\}\left(\phi^{2}+\zeta^{2}\right) .
\end{aligned}
$$

By the properties of the viscous contact wave, one can obtain

$\int_{\tau_{1}}^{\tau} f_{\mathbf{R}}\left(\left|\Theta_{y}^{C D}\right|^{2},\left|\Theta_{y y}^{C D}\right|\right)\left(\phi^{2}+\zeta^{2}\right) d y d \tau \leq C \delta \int_{\tau_{1}}^{\tau} f_{\mathbf{R}}(1+\tau)^{-1} e^{-\frac{c_{0} y^{2}}{1+\tau}}|(\phi, \zeta)|^{2} d y d \tau$,

while by the properties of the approximate rarefaction wave in Lemma 2.2, we have that for $i=1,3$,

$$
\begin{aligned}
& \int_{\tau_{1}}^{\tau} f_{\mathbf{R}}\left(\left|\left(V_{y}^{R_{i}}, U_{y}^{R_{i}}, \Theta_{y}^{R_{i}}\right)\right|^{2},\left|\Theta_{y y}^{R_{i}}\right|\right)\left(\phi^{2}+\zeta^{2}\right) d y d \tau \\
& \leq \int_{\tau_{1}}^{\tau}\left(\left\|\left(V_{y}^{R_{i}}, U_{y}^{R_{i}}, \Theta_{y}^{R_{i}}\right)\right\|^{2}+\left\|\Theta_{y y}^{R_{i}}\right\|_{L^{1}}\right)\|(\phi, \zeta)\|_{L^{\infty}}^{2} d \tau
\end{aligned}
$$




$$
\begin{aligned}
& \leq C \int_{\tau_{1}}^{\tau}(1+\tau)^{-1}\|(\phi, \zeta)\|\left\|\left(\phi_{y}, \zeta_{y}\right)\right\| d \tau \\
& \leq \mu \int_{\tau_{1}}^{\tau}\left\|\left(\phi_{y}, \zeta_{y}\right)\right\|^{2} d \tau+C_{\mu} \int_{\tau_{1}}^{\tau}(1+\tau)^{-2}\|(\phi, \zeta)\|^{2} d \tau,
\end{aligned}
$$

where and in the sequel $\mu$ is a small positive constant to be determined and $C_{\mu}$ is some positive constant depending on $\mu$.

Now, it remains to estimate the terms $Q_{1} \psi, Q_{2} \frac{\zeta}{\theta}$ on the right-hand side of (3.8) and the term $\left|Q_{2}\right|\left(\phi^{2}+\zeta^{2}\right)$ on the right-hand side of (3.11). For simplicity, we only estimate $Q_{2} \frac{\zeta}{\theta}$. By $(2.20)$, we find that

$$
\begin{aligned}
& \int_{\tau_{1}}^{\tau} f_{\mathbf{R}}\left|Q_{2} \frac{\zeta}{\theta}\right| d y d \tau \leq C \int_{\tau_{1}}^{\tau}\|\zeta\|_{L_{y}^{\infty}}\left\|Q_{2}\right\|_{L_{y}^{1}} d \tau \\
& \quad \leq C \int_{\tau_{1}}^{\tau}\|\zeta\|^{\frac{1}{2}}\left\|\zeta_{y}\right\|^{\frac{1}{2}}\left(\left\|Q_{21}\right\|_{L_{y}^{1}}+\left\|Q_{22}\right\|_{L_{y}^{1}}+\left\|Q^{C D}\right\|_{L_{y}^{1}}\right) d \tau \\
& \quad \leq C \int_{\tau_{1}}^{\tau}\|\zeta\|^{\frac{1}{2}}\left\|\zeta_{y}\right\|^{\frac{1}{2}}\left(\delta e^{-C(1+\tau)}+\left(\delta^{r_{1}}+\delta^{r_{3}}\right)^{\frac{1}{8}}(1+\tau)^{-\frac{7}{8}}+\delta(1+\tau)^{-\frac{3}{2}}\right) d \tau \\
& \quad \leq \mu \int_{\tau_{1}}^{\tau}\left\|\zeta_{y}\right\|^{2} d \tau+C_{\mu} \delta^{\frac{1}{6}} \int_{\tau_{1}}^{\tau}\|\zeta\|^{\frac{2}{3}}(1+\tau)^{-\frac{7}{6}} d \tau \\
& \quad \leq \mu \int_{\tau_{1}}^{\tau}\left\|\zeta_{y}\right\|^{2} d \tau+C_{\mu} \int_{\tau_{1}}^{\tau}\|\zeta\|^{2}(1+\tau)^{-\frac{7}{6}} d \tau+C_{\mu} \delta^{\frac{1}{4}} .
\end{aligned}
$$

Similarly, one can control the term $Q_{1} \psi$ and $\left|Q_{2}\right|\left(\phi^{2}+\zeta^{2}\right)$. Thus, substituting all the above estimates into (3.8) and choosing $\mu$ in the front of the integral $\int_{\tau_{1}}^{\tau}\left\|\left(\psi_{y}, \zeta_{y}\right)\right\|^{2} d \tau$ small enough, so that the integral can be absorbed by the left-hand side of (3.8), one concludes

$$
\begin{aligned}
& \|(\phi, \psi, \zeta)(\tau, \cdot)\|^{2}+\int_{\tau_{1}}^{\tau}\left\{\left\|\left(\psi_{y}, \zeta_{y}\right)(\tau, \cdot)\right\|^{2}+\left\|\sqrt{\left(U_{y}^{R_{1}}, U_{y}^{R_{3}}\right)}(\phi, \zeta)(\tau, \cdot)\right\|^{2}\right\} d \tau \\
& \leq C\left\|(\phi, \psi, \zeta)\left(\tau_{1}, \cdot\right)\right\|^{2}+C \int_{\tau_{1}}^{\tau}(1+\tau)^{-\frac{7}{6}}\|(\phi, \psi, \zeta)\|^{2} d \tau+C \delta^{\frac{1}{4}} \\
& +C \mu \int_{\tau_{1}}^{\tau}\left\|\phi_{y}(\tau, \cdot)\right\|^{2} d \tau+C \delta \int_{\tau_{1}}^{\tau} f_{\mathbf{R}}(1+\tau)^{-1} e^{-\frac{c_{0} y^{2}}{1+\tau}}|(\phi, \zeta)|^{2} d y d \tau .
\end{aligned}
$$

Next, we estimate $\left\|\phi_{y}\right\|^{2}$. Denote $\tilde{v}=\frac{v}{V}$. From the system $(3.1)_{2}$, one has

$$
\left(\frac{\tilde{v}_{y}}{\tilde{v}}\right)_{\tau}-\psi_{\tau}-(p-P)_{y}-Q_{1}=0 .
$$


Multiplying the above equation by $\frac{\tilde{v}_{y}}{\tilde{v}}$ and noticing that

$$
-(p-P)_{y}=\frac{R \theta}{v} \frac{\tilde{v}_{y}}{\tilde{v}}-\frac{R \zeta_{y}}{v}+(p-P) \frac{V_{y}}{V}-R \Theta_{y}\left(\frac{1}{V}-\frac{1}{v}\right),
$$

one obtains

$$
\begin{aligned}
& \left(\frac{1}{2}\left(\frac{\tilde{v}_{y}}{\tilde{v}}\right)^{2}-\psi \frac{\tilde{v}_{y}}{\tilde{v}}\right)_{\tau}+\left(\psi \frac{\tilde{v}_{\tau}}{\tilde{v}}\right)_{y}+\frac{R \theta}{v}\left(\frac{\tilde{v}_{y}}{\tilde{v}}\right)^{2} \\
& \quad=\psi_{y}\left(\frac{u_{y}}{v}-\frac{U_{y}}{V}\right)+\left(\frac{R \zeta_{y}}{v}-(p-P) \frac{V_{y}}{V}+R \Theta_{y}\left(\frac{1}{V}-\frac{1}{v}\right)-Q_{1}\right) \frac{\tilde{v}_{y}}{\tilde{v}} .
\end{aligned}
$$

Integrating the above equality with respect to $y$ and $\tau$ over $\mathbf{R}^{ \pm} \times\left[\tau_{1}, \tau\right]$ and using Cauchy-Schwarz's inequality, we infer that

$$
\begin{aligned}
& f_{\mathbf{R}}\left(\frac{1}{2}\left(\frac{\tilde{v}_{y}}{\tilde{v}}\right)^{2}-\psi \frac{\tilde{v}_{y}}{\tilde{v}}\right)(\tau, y) d y+\int_{\tau_{1}}^{\tau}\left[\psi \frac{\tilde{v}_{\tau}}{\tilde{v}}\right](\tau) d \tau+\int_{\tau_{1}}^{\tau} f_{\mathbf{R}} \frac{R \theta}{2 v}\left(\frac{\tilde{v}_{y}}{\tilde{v}}\right)^{2} d y d \tau \\
\leq & f_{\mathbf{R}}\left(\frac{1}{2}\left(\frac{\tilde{v}_{y}}{\tilde{v}}\right)^{2}-\psi \frac{\tilde{v}_{y}}{\tilde{v}}\right)\left(\tau_{1}, y\right) d y+\int_{\tau_{1}}^{\tau} f_{\mathbf{R}}\left|\psi_{y}\left(\frac{u_{y}}{v}-\frac{U_{y}}{V}\right)\right| d y d \tau \\
& +C \int_{\tau_{1}}^{\tau} f_{\mathbf{R}}\left|\frac{R \zeta_{y}}{v}-(p-P) \frac{V_{y}}{V}+R \Theta_{y}\left(\frac{1}{V}-\frac{1}{v}\right)-Q_{1}\right|^{2} d y d \tau,
\end{aligned}
$$

where the jump across $y=0$ can be bounded as follows.

$$
\begin{aligned}
& \int_{\tau_{1}}^{\tau}\left[\psi \frac{\tilde{v}_{\tau}}{\tilde{v}}\right](\tau) d \tau=\int_{\tau_{1}}^{\tau} \psi(\tau, 0)\left[\frac{u_{y}}{v}-\frac{U_{y}}{V}\right](\tau) d \tau=\int_{\tau_{1}}^{\tau} \psi(\tau, 0)[p](\tau) d \tau \\
& \quad=R \int_{\tau_{1}}^{\tau} \psi(\tau, 0) \theta(\tau, 0)\left[\frac{1}{v}\right](\tau) d \tau=-R \int_{\tau_{1}}^{\tau} \frac{\psi(\tau, 0) \theta(\tau, 0)}{v(\tau, 0+) v(\tau, 0-)}[v](\tau) d \tau \\
& \quad \leq C \int_{\tau_{1}}^{\tau}\|\psi\|_{L^{\infty}}(\tau)|[v]|\left(\tau_{1}\right) e^{-C\left(\tau-\tau_{1}\right)} d \tau \leq C \delta \int_{\tau_{1}}^{\tau}\|\psi\|^{\frac{1}{2}}\left\|\psi_{y}\right\|^{\frac{1}{2}} e^{-C\left(\tau-\tau_{1}\right)} d \tau \\
& \quad \leq \delta \int_{\tau_{1}}^{\tau}\left\|\psi_{y}\right\|^{2} d \tau+\delta \sup _{\tau \in\left[\tau_{1}, \tau_{2}\right]}\|\psi\|^{2}(\tau)+C \delta .
\end{aligned}
$$

Using the equality

$$
\frac{\tilde{v}_{y}}{\tilde{v}}=\frac{v_{y}}{v}-\frac{V_{y}}{V}=\frac{\phi_{y}}{v}-\frac{V_{y} \phi}{v V}
$$

we see that

$$
C^{-1}\left(\left|\phi_{y}\right|^{2}-\left|V_{y} \phi\right|^{2}\right) \leq\left(\frac{\tilde{v}_{y}}{\tilde{v}}\right)^{2} \leq C\left(\left|\phi_{y}\right|^{2}+\left|V_{y} \phi\right|^{2}\right) .
$$


From the definition of $Q_{1}$ in (2.19) it follows that

$$
\begin{aligned}
& \int_{\tau_{1}}^{\tau}\left\|Q_{1}\right\|^{2} d \tau \leq C \int_{\tau_{1}}^{\tau}\left(\left\|Q_{11}\right\|^{2}+\left\|Q_{12}\right\|^{2}\right) d \tau \\
& \quad \leq C \int_{\tau_{1}}^{\tau}\left(\left\|Q_{11}\right\|^{2}+\left\|\left(U_{y y}^{R_{1}}, U_{y y}^{R_{3}}, U_{y}^{R_{1}} V_{y}^{R_{1}}, U_{y}^{R_{3}} V_{y}^{R_{3}}\right)\right\|^{2}\right) d \tau \leq C \delta^{\frac{1}{4}} .
\end{aligned}
$$

Therefore, substituting all the above estimates into (3.13), we conclude that

$$
\begin{aligned}
& \left\|\phi_{y}(\tau, \cdot) H^{2}+\int_{\tau_{1}}^{\tau}\right\| \phi_{y} H^{2} d \tau \leq C\left\|\left(\phi, \psi, \phi_{y}\right) H^{2}\left(\tau_{1}\right)+C\right\|(\phi, \psi)(\tau, \cdot) \|^{2} \\
+ & C \delta \int_{\tau_{1}}^{\tau} f_{\mathbf{R}}(1+\tau)^{-1} e^{-\frac{c_{0} y^{2}}{1+\tau}}|(\phi, \zeta)|^{2} d y d \tau+C \int_{\tau_{1}}^{\tau}\left\|\left(\psi_{y}, \zeta_{y}\right)\right\|^{2} d \tau \\
+ & C \int_{\tau_{1}}^{\tau}(1+\tau)^{-\frac{7}{6}}\|(\phi, \psi, \zeta)\|^{2} d \tau+C \delta^{\frac{1}{4}}
\end{aligned}
$$

Multiplying the inequality (3.12) by a large constant $C_{1}>0$, and summing the resulting inequality with (3.14), we obtain Lemma 3.4. This completes the proof.

Next, we derive the higher order estimates, which are summarized in the following Lemma:

Lemma 3.5. Under the assumptions of Proposition 3.3, it holds that

$$
\begin{aligned}
& N\left(\tau_{1}, \tau_{2}\right) \\
& \quad+\int_{\tau_{1}}^{\tau_{2}}\left\{\left\|\sqrt{\left(U_{y}^{R_{1}}, U_{y}^{R_{3}}\right)}(\phi, \zeta)\right\|^{2}+\left\|\phi_{y}\right\|^{2}+\left\|\left(\psi_{y}, \zeta_{y}\right) H_{1}^{2}+\right\|\left(\psi_{y \tau}, \zeta_{y \tau}\right) \|^{2}\right\} d \tau \\
& \leq C N\left(\tau_{1}\right)+C \int_{\tau_{1}}^{\tau_{2}}(1+\tau)^{-\frac{7}{6}}\|(\phi, \psi, \zeta)\|^{2} d \tau+C \delta^{\frac{1}{4}} \\
& \quad+C \delta \int_{\tau_{1}}^{\tau_{2}} f_{\mathbf{R}}(1+\tau)^{-1} e^{-\frac{c_{0} y^{2}}{1+\tau}}|(\phi, \zeta)|^{2} d y d \tau .
\end{aligned}
$$

Proof: Multiplying the equation $(3.1)_{2}$ by $-\psi_{y y}$, one gets

$$
\left(\frac{\psi_{y}^{2}}{2}\right)_{\tau}-\left(\psi_{\tau} \psi_{y}\right)_{y}+\frac{\psi_{y y}^{2}}{v}=\left\{(p-P)_{y}+\frac{v_{y}}{v^{2}} \psi_{y}-\left(U_{y}\left(\frac{1}{v}-\frac{1}{V}\right)\right)_{y}+Q_{1}\right\} \psi_{y y} .
$$

Integration of the above equation with respect to $y$ and $\tau$ over $\mathbf{R}^{ \pm} \times\left[\tau_{1}, \tau\right]$ 
gives

$$
\begin{aligned}
& f_{\mathbf{R}} \frac{\psi_{y}^{2}}{2}(\tau, y) d y+\int_{\tau_{1}}^{\tau} f_{\mathbf{R}} \frac{\psi_{y y}^{2}}{v} d y d \tau=f_{\mathbf{R}} \frac{\psi_{y}^{2}}{2}\left(\tau_{1}, y\right) d y-\int_{\tau_{1}}^{\tau}\left[\psi_{\tau} \psi_{y}\right](\tau) d \tau \\
&+\int_{\tau_{1}}^{\tau} f_{\mathbf{R}}\left\{(p-P)_{y}+\frac{v_{y}}{v^{2}} \psi_{y}-\left(U_{y}\left(\frac{1}{v}-\frac{1}{V}\right)\right)_{y}+Q_{1}\right\} \psi_{y y} d y d \tau=: \sum_{i=1}^{3} J_{i} .
\end{aligned}
$$

We only have to estimate $J_{i}(i=2,3)$. First, the jump $J_{2}$ can be bounded as follows.

$$
\begin{aligned}
J_{2} & =-\int_{\tau_{1}}^{\tau}\left[\psi_{\tau} \psi_{y}\right](\tau) d \tau=-\int_{\tau_{1}}^{\tau} \psi_{\tau}(\tau, 0)\left[\psi_{y}\right](\tau) d \tau \\
& =-\int_{\tau_{1}}^{\tau} \psi_{\tau}(\tau, 0)\left[u_{y}\right](\tau) d \tau=-\int_{\tau_{1}}^{\tau} \psi_{\tau}(\tau, 0)\left[\left(\frac{u_{y}}{v}-p\right) v\right](\tau) d \tau \\
& =-\int_{\tau_{1}}^{\tau} \psi_{\tau}(\tau, 0)\left(\frac{u_{y}}{v}-p\right)(\tau, 0)[v](\tau) d \tau \\
& \leq C \int_{\tau_{1}}^{\tau}\left\|\psi_{\tau}\right\|_{L^{\infty}}\left(\left\|\psi_{y}\right\|_{L^{\infty}}+1\right)[v]\left(\tau_{1}\right) e^{-C\left(\tau-\tau_{1}\right)} d \tau \\
& \leq C \delta \int_{\tau_{1}}^{\tau}\left\|\psi_{\tau}\right\|^{\frac{1}{2}}\left\|\psi_{y \tau}\right\|^{\frac{1}{2}}\left(\left\|\psi_{y}\right\|^{\frac{1}{2}}\left\|\psi_{y y}\right\|^{\frac{1}{2}}+1\right) e^{-C\left(\tau-\tau_{1}\right)} d \tau
\end{aligned}
$$

In view of $(3.1)_{2}$ and (3.3), one has

$$
\begin{aligned}
\left\|\psi_{\tau}\right\| & \leq C\left(\left\|\psi_{y y} \#+\right\|\left(\phi_{y}, \psi_{y}, \zeta_{y}\right) \#+\left\|\left(U_{y y}, V_{y}, U_{y}, \Theta_{y}\right) \phi\right\|+\left\|Q_{1}\right\|\right) \\
& \leq C\left(\left\|\psi_{y y} \#+\right\|\left(\phi_{y}, \psi_{y}, \zeta_{y}\right) \#+\delta\right)
\end{aligned}
$$

Substituting (3.17) into (3.16), we obtain

$$
\begin{aligned}
\left|J_{2}\right| \leq & C \delta \int_{\tau_{1}}^{\tau}\left(\left\|\psi_{y y} \#+\right\|\left(\phi_{y}, \psi_{y}, \zeta_{y}\right) \#+\delta\right)^{\frac{1}{2}} \\
& \times \| \psi_{y \tau} \#^{\frac{1}{2}}\left(\left\|\psi_{y}\right\|^{\frac{1}{2}}\left\|\psi_{y y}\right\|^{\frac{1}{2}}+1\right) e^{-C\left(\tau-\tau_{1}\right)} d \tau \\
\leq & \mu \int_{\tau_{1}}^{\tau}\left\|\left(\psi_{y y}, \psi_{y \tau}\right) H^{2} d \tau+C_{\mu} \delta \int_{\tau_{1}}^{\tau}\right\|\left(\phi_{y}, \psi_{y}, \zeta_{y}\right) H^{2} d \tau+C_{\mu} \delta .
\end{aligned}
$$


On the other hand, $J_{3}$ can be estimates as follows.

$$
\begin{aligned}
J_{3}= & \int_{\tau_{1}}^{\tau} f_{\mathbf{R}}\left\{(p-P)_{y}+\frac{v_{y}}{v^{2}} \psi_{y}-\left(U_{y}\left(\frac{1}{v}-\frac{1}{V}\right)\right)_{y}+Q_{1}\right\} \psi_{y y} d y d \tau \\
\leq & C \int_{\tau_{1}}^{\tau} f_{\mathbf{R}}\left\{\left|\left(\phi_{y}, \zeta_{y}\right)\right|+|(\phi, \zeta)|\left|\left(\phi_{y}, V_{y}, \Theta_{y}, U_{y y}\right)\right|\right. \\
& \left.\quad+\left|\left(\phi_{y}, V_{y}\right)\right|\left|\left(\psi_{y}, U_{y}, U_{y} \phi\right)\right|+\left|Q_{1}\right|\right\}\left|\psi_{y y}\right| d y d \tau \\
\leq & \mu \int_{\tau_{1}}^{\tau}\left\|\psi_{y y}\right\|^{2} d \tau+C_{\mu} \int_{\tau_{1}}^{\tau} \|\left(\phi_{y}, \psi_{y}, \zeta_{y}\right) H^{2} d \tau \\
& +C_{\mu} \int_{\tau_{1}}^{\tau}(1+\tau)^{-\frac{7}{6}}\|(\phi, \psi, \zeta)\|^{2} d \tau \\
& +C_{\mu} \delta+C_{\mu} \delta \int_{\tau_{1}}^{\tau} f_{\mathbf{R}}(1+\tau)^{-1} e^{-\frac{c_{0} y^{2}}{1+\tau}}|(\phi, \zeta)|^{2} d y d \tau .
\end{aligned}
$$

Substituting (3.18) and (3.19) into (3.15) and choosing $\mu$ suitably small in the front of the integral $\int_{\tau_{1}}^{\tau} \| \psi_{y y} H^{2} d \tau$, we deduce that

$$
\begin{aligned}
& \left\|\psi_{y}\right\|^{2}(\tau)+\int_{\tau_{1}}^{\tau}\left\|\psi_{y y} H^{2} d \tau \leq C\right\| \psi_{y}\left\|^{2}\left(\tau_{1}\right)+C \mu \int_{\tau_{1}}^{\tau}\right\| \psi_{y \tau} H^{2} d \tau \\
& \quad+C_{\mu} \int_{\tau_{1}}^{\tau}(1+\tau)^{-\frac{7}{6}}\|(\phi, \zeta)\|^{2} d \tau+C_{\mu} \delta+C_{\mu} \int_{\tau_{1}}^{\tau} \|\left(\phi_{y}, \psi_{y}, \zeta_{y}\right) H^{2} d \tau \\
& \quad+C_{\mu} \delta \int_{\tau_{1}}^{\tau} f_{\mathbf{R}}(1+\tau)^{-1} e^{-\frac{c_{0} y^{2}}{1+\tau}}|(\phi, \zeta)|^{2} d y d \tau .
\end{aligned}
$$

Multiplication of the equation $(3.1)_{3}$ with $-\zeta_{y y}$ yields that

$$
\begin{aligned}
& \frac{R}{\gamma-1}\left(\frac{\zeta_{y}^{2}}{2}\right)_{\tau}-\frac{R}{\gamma-1}\left(\zeta_{\tau} \zeta_{y}\right)_{y}+\nu \frac{\zeta_{y y}^{2}}{v} \\
& =\left\{\left(p u_{y}-P U_{y}\right)+\nu \frac{\zeta_{y} v_{y}}{v^{2}}-\nu\left(\Theta_{y}\left(\frac{1}{v}-\frac{1}{V}\right)\right)_{y}-\left(\frac{u_{y}^{2}}{v}-\frac{U_{y}^{2}}{V}\right)+Q_{2}\right\} \zeta_{y y} .
\end{aligned}
$$

Integrating the above equality with respect to $y$ and $\tau$ over $\mathbf{R}^{ \pm} \times\left[\tau_{1}, \tau\right]$, and employing almost the same arguments as those used for $\left\|\psi_{y}\right\|^{2}(\tau)$ in (3.20), we obtain

$$
\left\|\zeta_{y}\right\|^{2}(\tau)+\int_{\tau_{1}}^{\tau}\left\|\zeta_{y y}\right\|^{2} d \tau \leq C\left\|\zeta_{y}\right\|^{2}\left(\tau_{1}\right)+C \delta^{\frac{1}{4}}+C \int_{\tau_{1}}^{\tau}(1+\tau)^{-\frac{7}{6}}\|(\phi, \zeta)\|^{2} d \tau
$$




$$
+C \int_{\tau_{1}}^{\tau} \|\left(\phi_{y}, \psi_{y}, \zeta_{y}\right) H^{2} d \tau+C(\delta)^{2} \int_{\tau_{1}}^{\tau} f_{\mathbf{R}}(1+\tau)^{-1} e^{-\frac{c_{0} y^{2}}{1+\tau}}|(\phi, \zeta)|^{2} d y d \tau
$$

where we have used the following jump estimate across $y=0$

$$
\begin{aligned}
& -\frac{R}{\gamma-1} \int_{\tau_{1}}^{\tau}\left[\zeta_{\tau} \zeta_{y}\right](\tau) d \tau=-\frac{R}{\gamma-1} \int_{\tau_{1}}^{\tau} \zeta_{\tau}(\tau, 0)\left[\zeta_{y}\right](\tau) d \tau \\
& =-\frac{R}{\gamma-1} \int_{\tau_{1}}^{\tau} \zeta_{\tau}(\tau, 0)\left[\theta_{y}\right](\tau) d \tau=-\frac{R}{\gamma-1} \int_{\tau_{1}}^{\tau} \zeta_{\tau}(\tau, 0) \frac{\theta_{y}}{v}(\tau, 0)[v](\tau) d \tau \\
& \leq C \int_{\tau_{1}}^{\tau}\left\|\zeta_{\tau}\right\|_{L^{\infty}}\left(1+\left\|\zeta_{y}\right\|_{L^{\infty}}\right)[v]\left(\tau_{1}\right) e^{-C\left(\tau-\tau_{1}\right)} d \tau \\
& \leq C \delta \int_{\tau_{1}}^{\tau}\left\|\zeta_{\tau} \#^{\frac{1}{2}}\right\| \zeta_{y \tau} \#^{\frac{1}{2}}\left(1+\left\|\zeta_{y}\right\|^{\frac{1}{2}} \| \zeta_{y y} \#^{\frac{1}{2}}\right) e^{-C\left(\tau-\tau_{1}\right)} d \tau
\end{aligned}
$$

and the estimate

$$
\begin{aligned}
\left\|\zeta_{\tau}\right\| & \leq C\left(\left\|\zeta_{y y} \#+\right\|\left(\phi_{y}, \psi_{y}, \zeta_{y}\right) \#+\left\|\left(U_{y}, \Theta_{y y}, \Theta_{y} V_{y}, U_{y}^{2}\right)(\phi, \zeta)\right\|+\left\|Q_{2}\right\|\right) \\
& \leq C\left(\left\|\zeta_{y y} \#+\right\|\left(\phi_{y}, \psi_{y}, \zeta_{y}\right) \#+\delta\right) .
\end{aligned}
$$

It follows from (3.17) and (3.22) that

$$
\begin{gathered}
\int_{\tau_{1}}^{\tau_{2}}\left\|\left(\psi_{\tau}, \zeta_{\tau}\right)(\tau, \cdot)\right\|^{2} d \tau \\
\leq C\left(\int_{\tau_{1}}^{\tau_{2}}\left\|\left(\psi_{y y}, \zeta_{y y}\right)\right\|^{2} d \tau+\int_{\tau_{1}}^{\tau_{2}}\left\|\left(\phi_{y}, \psi_{y}, \zeta_{y}\right)\right\|^{2} d \tau\right. \\
\left.\quad+\int_{\tau_{1}}^{\tau_{2}}\left\|\left(U_{y}, \Theta_{y y}, \Theta_{y} V_{y}, U_{y}^{2}\right)(\phi, \zeta)\right\|^{2} d \tau+\int_{\tau_{1}}^{\tau_{2}}\left\|Q_{2}\right\|^{2} d \tau\right) \\
\leq C \int_{\tau_{1}}^{\tau_{2}}\left\|\left(\psi_{y y}, \zeta_{y y}\right)\right\|^{2} d \tau+C \int_{\tau_{1}}^{\tau_{2}}\left\|\left(\phi_{y}, \psi_{y}, \zeta_{y}\right)\right\|^{2} d \tau \\
\quad+\int_{\tau_{1}}^{\tau_{2}}(1+\tau)^{-2}\|(\phi, \zeta)\|^{2} d \tau+C \delta^{\frac{1}{4}}
\end{gathered}
$$

Now we turn to control $\sup _{\tau \in\left[\tau_{1}, \tau_{2}\right]} \|\left(\psi_{\tau}, \zeta_{\tau}\right) \mathbb{H}^{2}$. First, applying the operator $\partial_{\tau}$ to the equation $(3.1)_{2}$, we get

$$
\psi_{\tau \tau}=\left(\frac{u_{y}}{v}-p\right)_{y \tau}-\left(\frac{U_{y}}{V}-P\right)_{y \tau}-Q_{1 \tau}
$$


Multiplication of the above equation by $\psi_{\tau}$ gives

$$
\begin{aligned}
& \left(\frac{\psi_{\tau}^{2}}{2}\right)_{\tau}+\frac{\psi_{y \tau}^{2}}{v}=\left\{\psi_{\tau}\left(\frac{u_{y}}{v}-p\right)_{\tau}-\psi_{\tau}\left(\frac{U_{y}}{V}-P\right)_{\tau}\right\}_{y} \\
& \quad-\psi_{y \tau} \frac{U_{y \tau}}{v}+\psi_{y \tau} \frac{u_{y}}{v^{2}} v_{\tau}+\psi_{y \tau}\left(\frac{U_{y}}{V}\right)_{\tau}+\psi_{y \tau}(p-P)_{\tau}-\psi_{\tau} Q_{1 \tau}
\end{aligned}
$$

If we integrate the above equality with respect to $y$ and $\tau$ over $\mathbf{R}^{ \pm} \times\left[\tau_{1}, \tau\right]$, we find that

$$
\begin{aligned}
& f_{\mathbf{R}} \frac{\psi_{\tau}^{2}}{2}(\tau, y) d y+\int_{\tau_{1}}^{\tau} f_{\mathbf{R}} \frac{\psi_{y \tau}^{2}}{v} d y d \tau \\
& \quad=f_{\mathbf{R}} \frac{\psi_{\tau}^{2}}{2}\left(\tau_{1}, y\right) d y-\int_{\tau_{1}}^{\tau}\left[\psi_{\tau}\left(\frac{u_{y}}{v}-p\right)_{\tau}-\psi_{\tau}\left(\frac{U_{y}}{V}-P\right)_{\tau}\right](\tau) d \tau \\
& +\int_{\tau_{1}}^{\tau} f_{\mathbf{R}}\left\{-\psi_{y \tau} \frac{U_{y \tau}}{v}+\psi_{y \tau} \frac{u_{y}}{v^{2}} v_{\tau}+\psi_{y \tau}\left(\frac{U_{y}}{V}\right)_{\tau}+\psi_{y \tau}(p-P)_{\tau}-\psi_{\tau} Q_{1 \tau}\right\} d y d \tau
\end{aligned}
$$

where the jump across $y=0$ in fact vanishes, i.e.,

$$
\begin{aligned}
& {\left[\psi_{\tau}\left(\frac{u_{y}}{v}-p\right)_{\tau}-\psi_{\tau}\left(\frac{U_{y}}{V}-P\right)_{\tau}\right](\tau)} \\
& =\left[\psi_{\tau}\right](\tau)\left(\frac{u_{y}}{v}-p\right)_{\tau}(\tau, 0-) \\
& \quad+\psi_{\tau}(\tau, 0+)\left[\left(\frac{u_{y}}{v}-p\right)_{\tau}\right](\tau)-\left[\psi_{\tau}\right](\tau)\left(\frac{U_{y}}{V}-P\right)_{\tau}(\tau, 0) \\
& =[\psi]_{\tau}(\tau)\left(\frac{u_{y}}{v}-p\right)_{\tau}(\tau, 0-) \\
& \quad+\psi_{\tau}(\tau, 0+)\left[\frac{u_{y}}{v}-p\right]_{\tau}(\tau)-[\psi]_{\tau}(\tau)\left(\frac{U_{y}}{V}-P\right)_{\tau}(\tau, 0) \\
& =0
\end{aligned}
$$

Now we apply $\partial_{\tau}$ to the equation $(3.1)_{3}$ to deduce that

$$
\frac{R}{\gamma-1} \zeta_{\tau \tau}=\nu\left(\frac{\theta_{y}}{v}\right)_{y \tau}-\nu\left(\frac{\Theta_{y}}{V}\right)_{y \tau}+\left\{u_{y}\left(\frac{u_{y}}{v}-p\right)\right\}_{\tau}-\left\{u_{y}\left(\frac{U_{y}}{V}-P\right)\right\}_{\tau}-Q_{2 \tau}
$$

Multiplying the above equation by $\zeta_{\tau}$, one has

$$
\frac{R}{\gamma-1}\left(\frac{\zeta_{\tau}^{2}}{2}\right)_{\tau}+\nu \frac{\zeta_{y \tau}^{2}}{v}=\left\{\nu \zeta_{\tau}\left(\frac{\theta_{y}}{v}\right)_{\tau}-\nu \zeta_{\tau}\left(\frac{\Theta_{y}}{V}\right)_{\tau}\right\}_{y}
$$




$$
\begin{aligned}
& +\nu \zeta_{y \tau} \frac{\Theta_{y \tau}}{v}+\nu \zeta_{y \tau} \frac{\theta_{y}}{v^{2}} v_{\tau}+\nu \zeta_{y \tau}\left(\frac{\Theta_{y}}{V}\right)_{\tau}+\zeta_{\tau} u_{y \tau}\left(\frac{u_{y}}{v}-p\right) \\
& +\zeta_{\tau} u_{y}\left(\frac{u_{y}}{v}-p\right)_{\tau}-\zeta_{\tau} U_{y \tau}\left(\frac{U_{y}}{V}-P\right)-\zeta_{\tau} U_{y}\left(\frac{U_{y}}{V}-P\right)_{\tau}-\zeta_{\tau} Q_{2 \tau}
\end{aligned}
$$

Integrating the above equality with respect to $y$ and $\tau$ over $\mathbf{R}^{ \pm} \times\left[\tau_{1}, \tau\right]$, we deduce that

$$
\begin{aligned}
& f_{\mathbf{R}} \frac{R \zeta_{\tau}^{2}}{2(\gamma-1)}(\tau, y) d y+\int_{\tau_{1}}^{\tau} f_{\mathbf{R}} \nu \frac{\zeta_{y \tau}^{2}}{v} d y d \tau=f_{\mathbf{R}} \frac{R \zeta_{\tau}^{2}}{2(\gamma-1)}\left(\tau_{1}, y\right) d y \\
& -\int_{\tau_{1}}^{\tau}\left[\nu \zeta_{\tau}\left(\frac{\theta_{y}}{v}\right)_{\tau}-\nu \zeta_{\tau}\left(\frac{\Theta_{y}}{V}\right)_{\tau}\right](\tau) d \tau+\int_{\tau_{1}}^{\tau} f_{\mathbf{R}}\left\{\nu \zeta_{y \tau} \frac{\Theta_{y \tau}}{v}+\nu \zeta_{y \tau} \frac{\theta_{y}}{v^{2}} v_{\tau}\right. \\
& +\nu \zeta_{y \tau}\left(\frac{\Theta_{y}}{V}\right)_{\tau}+\zeta_{\tau} u_{y \tau}\left(\frac{u_{y}}{v}-p\right)+\zeta_{\tau} u_{y}\left(\frac{u_{y}}{v}-p\right)_{\tau}-\zeta_{\tau} U_{y \tau}\left(\frac{U_{y}}{V}-P\right) \\
& \left.-\zeta_{\tau} U_{y}\left(\frac{U_{y}}{V}-P\right)_{\tau}-\zeta_{\tau} Q_{2 \tau}\right\} d y d \tau
\end{aligned}
$$

where the jump in fact vanishes.

$$
\begin{aligned}
& {\left[\nu \zeta_{\tau}\left(\frac{\theta_{y}}{v}\right)_{\tau}-\nu \zeta_{\tau}\left(\frac{\Theta_{y}}{V}\right)_{\tau}\right](\tau)} \\
& =\nu\left[\zeta_{\tau}\right](\tau)\left(\frac{\theta_{y}}{v}\right)_{\tau}(\tau, 0-)+\nu \zeta_{\tau}(\tau, 0+)\left[\left(\frac{\theta_{y}}{v}\right)_{\tau}\right](\tau)-\nu\left[\zeta_{\tau}\right](\tau)\left(\frac{\Theta_{y}}{V}\right)_{\tau}(\tau, 0) \\
& =\nu[\zeta]_{\tau}(\tau)\left(\frac{\theta_{y}}{v}\right)_{\tau}(\tau, 0-)+\nu \zeta_{\tau}(\tau, 0+)\left[\frac{\theta_{y}}{v}\right]_{\tau}(\tau)-\nu[\zeta]_{\tau}(\tau)\left(\frac{\Theta_{y}}{V}\right)_{\tau}(\tau, 0) \\
& =0
\end{aligned}
$$

Hence, taking into account (3.25) and (3.27), we get from (3.24) and (3.26) that

$$
\begin{aligned}
& \left\|\left(\psi_{\tau}, \zeta_{\tau}\right) \#^{2}(\tau)+\int_{\tau_{1}}^{\tau}\right\|\left(\psi_{y \tau}, \zeta_{y \tau}\right) H^{2} d \tau \leq C \|\left(\psi_{\tau}, \zeta_{\tau}\right) H^{2}\left(\tau_{1}\right) \\
& +C \int_{\tau_{1}}^{\tau}\left\|\left(\psi_{\tau}, \zeta_{\tau}\right) H^{2} d \tau+C \int_{\tau_{1}}^{\tau}(1+\tau)^{-\frac{7}{6}}\right\|(\phi, \zeta) \|^{2} d \tau+C \delta \\
& +C \int_{\tau_{1}}^{\tau}\left\|\left(\phi_{y}, \psi_{y}, \zeta_{y}\right)\right\|^{2} d \tau+C \delta \int_{\tau_{1}}^{\tau} f_{\mathbf{R}}(1+\tau)^{-1} e^{-\frac{c_{0} y^{2}}{1+\tau}}|(\phi, \zeta)|^{2} d y d \tau .
\end{aligned}
$$

Combing the estimates (3.20), (3.21), (3.23), (3.28) and Lemma 3.4 together, we obtain Lemma 3.5, and the proof is completed. 
It remains to control the term

$$
\delta \int_{\tau_{1}}^{\tau} f_{\mathbf{R}}(1+\tau)^{-1} e^{-\frac{c_{0} y^{2}}{1+\tau}}|(\phi, \zeta)|^{2} d y d \tau
$$

which comes from the viscous contact wave. We shall use the estimate on the heat kernel in [15] to get the desired estimates.

Lemma 3.6. Suppose that $Z(t, y)$ satisfies

$Z \in L^{\infty}\left(0, T ; L^{2}\left(\mathbf{R}^{ \pm}\right)\right), \quad Z_{y} \in L^{2}\left(0, T ; L^{2}\left(\mathbf{R}^{ \pm}\right)\right), \quad Z_{\tau} \in L^{2}\left(0, T ; H^{-1}\left(\mathbf{R}^{ \pm}\right)\right)$,

then

$$
\begin{aligned}
& \int_{\tau_{1}}^{\tau} f_{\mathbf{R}}(1+\tau)^{-1} Z^{2} e^{-\frac{2 \beta y^{2}}{1+\tau}} d y d \tau \\
& \leq C_{\beta}\left\{\left\|Z\left(\tau_{1}, y\right)\right\|^{2}+\int_{\tau_{1}}^{\tau} \| h_{y} H^{2} d \tau+\int_{\tau_{1}}^{\tau}\left\langle Z_{\tau}, Z g_{\beta}^{2}\right\rangle_{H^{1} \times H^{-1}\left(\mathbf{R}^{ \pm}\right)} d \tau\right\}
\end{aligned}
$$

where

$$
g_{\beta}(\tau, y)=(1+\tau)^{-\frac{1}{2}} \int_{0}^{y} e^{-\frac{\beta \eta^{2}}{1+\tau}} d \eta
$$

and $\beta>0$ is the constant to be determined.

Remark 3.7. Lemma 3.6 can be shown using arguments similar to those in [15], and hence its proof will be omitted here for simplicity. Note that the domain considered here consists of two half lines $\mathbf{R}^{ \pm}$, and hence the jump across $y=0$ should be treated. In view of this, the functional $g_{\beta}$ should be chosen in (3.30), so that $g_{\beta}$ is continuous at $y=0$. Furthermore, it holds that $g_{\beta}(\tau, 0) \equiv 0$.

Lemma 3.8. Under the assumptions of Proposition 3.3, it holds that

$$
\begin{array}{r}
\int_{\tau_{1}}^{\tau} f_{\mathbf{R}} \frac{e^{-\frac{c_{0} y^{2}}{1+\tau}}}{1+\tau}|(\phi, \psi, \zeta)|^{2} d y d \tau \leq C \delta+C\left\|(\phi, \psi, \zeta)\left(\tau_{1}, \cdot\right)\right\|^{2}+C\|(\phi, \psi, \zeta)(\tau, \cdot)\|^{2} \\
+C \int_{\tau_{1}}^{\tau}\left\|\left(\phi_{y}, \psi_{y}, \zeta_{y}\right) H^{2} d \tau+C \int_{\tau_{1}}^{\tau}(1+\tau)^{-\frac{7}{6}}\right\|(\phi, \psi) \|^{2} d \tau .
\end{array}
$$

Proof: From the equation $(3.1)_{2}$ and the fact $p-P=\frac{R \zeta-P \phi}{v}$ one gets

$$
\psi_{\tau}+\left(\frac{R \zeta-P \phi}{v}\right)_{y}=\left(\frac{u_{y}}{v}-\frac{U_{y}}{V}\right)_{y}-Q_{1} .
$$


Let

$$
G_{\alpha}(\tau, y)=(1+\tau)^{-1} \int_{0}^{y} e^{-\frac{\alpha \eta^{2}}{1+\tau}} d \eta
$$

where $\alpha$ is a positive constant to be determined. Multiplying the equation (3.31) by $G_{\alpha}(R \zeta-P \phi)$, we find that

$$
\begin{aligned}
& \left(\frac{G_{\alpha}(R \zeta-P \phi)^{2}}{2 v}\right)_{y}-\left(G_{\alpha}\right)_{y} \frac{(R \zeta-P \phi)^{2}}{2 v}+\frac{G_{\alpha}(R \zeta-P \phi)^{2}}{2 v^{2}}\left(V_{y}+\phi_{y}\right) \\
& =-G_{\alpha}(R \zeta-P \phi) \psi_{\tau}+G_{\alpha}(R \zeta-P \phi)\left(\frac{u_{y}}{v}-\frac{U_{y}}{V}\right)_{y}-G_{\alpha}(R \zeta-P \phi) Q_{1} .
\end{aligned}
$$

Noticing that

$-G_{\alpha}(R \zeta-P \phi) \psi_{\tau}=-\left(G_{\alpha}(R \zeta-P \phi) \psi\right)_{\tau}+\left(G_{\alpha}\right)_{\tau}(R \zeta-P \phi) \psi+G_{\alpha} \psi(R \zeta-P \phi)_{\tau}$

and

$$
\begin{aligned}
& (R \zeta-P \phi)_{\tau}=R \zeta_{\tau}-P_{\tau} \phi-P \phi_{\tau} \\
& =-\gamma P \psi_{y}+(\gamma-1)\left\{-(p-P)\left(U_{y}+\psi_{y}\right)+\left(\frac{u_{y}^{2}}{v}-\frac{U_{y}^{2}}{V}\right)\right. \\
& \left.\quad+\nu\left(\frac{\theta_{y}}{v}-\frac{\Theta_{y}}{V}\right)_{y}-Q_{2}\right\}-P_{\tau} \phi
\end{aligned}
$$

if we insert (3.34) into (3.33) and use the equality

$$
-G_{\alpha} \gamma P \psi_{y} \psi=-\left(\gamma G_{\alpha} P \frac{\psi^{2}}{2}\right)_{y}+\gamma P\left(G_{\alpha}\right)_{y} \frac{\psi^{2}}{2}+\gamma P_{y} \frac{\psi^{2}}{2}
$$

we get from (3.32) that

(3.35) $\frac{e^{-\frac{\alpha y^{2}}{1+\tau}}}{2(1+\tau)}\left\{(R \zeta-P \phi)^{2}+\gamma P \psi^{2}\right\}=\left\{G_{\alpha} v(R \zeta-P \phi) \psi\right\}_{\tau}+H_{2 y}+Q_{4}$,

where

$$
\begin{aligned}
H_{2}= & \frac{G_{\alpha}(R \zeta-P \phi)^{2}}{2 v}+\gamma G_{\alpha} P \frac{\psi^{2}}{2}-\nu(\gamma-1) G_{\alpha} \psi\left(\frac{\theta_{y}}{v}-\frac{\Theta_{y}}{V}\right) \\
& -G_{\alpha}(R \zeta-P \phi)\left(\frac{u_{y}}{v}-\frac{U_{y}}{V}\right)
\end{aligned}
$$


and

$$
\begin{aligned}
Q_{4} & =\frac{G_{\alpha}(R \zeta-P \phi)^{2}}{2 v^{2}}\left(V_{y}+\phi_{y}\right)-\left(G_{\alpha}\right)_{\tau}(R \zeta-P \phi) \psi \\
& +\left(G_{\alpha}(R \zeta-P \phi)\right)_{y}\left(\frac{u_{y}}{v}-\frac{U_{y}}{V}\right) \\
& +(\gamma-1) G_{\alpha} \psi\left\{(p-P)\left(U_{y}+\psi_{y}\right)-\left(\frac{u_{y}^{2}}{v}-\frac{U_{y}^{2}}{V}\right)+Q_{2}\right\} \\
& +(\gamma-1) \nu\left(G_{\alpha} \psi\right)_{y}\left(\frac{\theta_{y}}{v}-\frac{\Theta_{y}}{V}\right)+G_{\alpha}(R \zeta-P \phi) Q_{1}+G_{\alpha} \psi P_{\tau} \phi-\gamma P_{y} \frac{\psi^{2}}{2}
\end{aligned}
$$

Integrating (3.35) over $\mathbf{R}^{ \pm} \times\left[\tau_{1}, \tau\right]$, one infers that

$$
\begin{array}{r}
\int_{\tau_{1}}^{\tau} f_{\mathbf{R}} \frac{e^{-\frac{\alpha y^{2}}{1+\tau}}}{1+\tau}\left\{(R \zeta-P \phi)^{2}+\psi^{2}\right\} d y d \tau=f_{\mathbf{R}}\left\{G_{\alpha} v(R \zeta-P \phi) \psi\right\}(\tau, y) d y \\
-f_{\mathbf{R}}\left\{G_{\alpha} v(R \zeta-P \phi) \psi\right\}\left(\tau_{1}, y\right) d y+\int_{\tau_{1}}^{\tau}\left[H_{2}\right](\tau) d \tau+\int_{\tau_{1}}^{\tau} f_{\mathbf{R}} Q_{4} d y d \tau .
\end{array}
$$

Here we only analyze the jump term $\left[H_{2}\right]$ across $y=0$, the other terms in (3.36) can be estimated similarly to those in [15] or [17]. Recalling that $G_{\alpha}(\tau, y)$ is continuous at $y=0$ and $G_{\alpha}(\tau, 0) \equiv 0$, we easily see that

$$
\left[H_{2}\right](\tau)=0
$$

Thus, from (3.36) one gets

$$
\begin{aligned}
\int_{\tau_{1}}^{\tau} f_{\mathbf{R}} \frac{e^{-\frac{\alpha y^{2}}{1+\tau}}}{1+\tau}\left\{(R \zeta-P \phi)^{2}+\psi^{2}\right\} d y d \tau \leq C \delta+C\left\|(\phi, \psi, \zeta)\left(\tau_{1}, \cdot\right)\right\|^{2} \\
+C\|(\phi, \psi, \zeta)(\tau, \cdot)\|^{2}+C \int_{\tau_{1}}^{\tau}(1+\tau)^{-\frac{7}{6}}\|(\phi, \psi, \zeta)(\tau, \cdot)\|^{2} d \tau \\
+C \int_{\tau_{1}}^{\tau}\left\|\left(\phi_{y}, \psi_{y}, \zeta_{y}\right)(\tau, \cdot)\right\|^{2} d \tau+C \delta \int_{\tau_{1}}^{\tau} f_{\mathbf{R}} \frac{e^{-\frac{\alpha y^{2}}{1+\tau}}}{1+\tau}|(\phi, \zeta)|^{2} d y d \tau
\end{aligned}
$$

In order to get the desired estimate in Lemma 3.8, we will use Lemma 3.6 to derive another similar estimate from the energy equation $(3.1)_{3}$. To this end, we set

$$
Z=\frac{R}{\gamma-1} \zeta+P \phi
$$


in Lemma 3.6. Thus we only need to compute the last term in (3.29). From the energy equation $(3.1)_{3}$, we have

$$
Z_{\tau}=P_{\tau} \phi-(p-P) u_{y}+\nu\left(\frac{\theta_{y}}{v}-\frac{\Theta_{y}}{V}\right)_{y}+\left(\frac{u_{y}^{2}}{v}-\frac{U_{y}^{2}}{V}\right)-Q_{2},
$$

whence

$$
\begin{aligned}
& \int_{\tau_{1}}^{\tau}\left\langle Z_{\tau}, Z g_{\beta}^{2}\right\rangle_{H^{1} \times H^{-1}\left(\mathbf{R}^{ \pm}\right)} d \tau \\
= & \int_{\tau_{1}}^{\tau} f_{\mathbf{R}}\left(P_{\tau} \phi-(p-P) U_{y}\right) Z g_{\beta}^{2} d y d \tau-\int_{\tau_{1}}^{\tau} f_{\mathbf{R}}(p-P) \psi_{y} Z g_{\beta}^{2} d y d \tau \\
& +\int_{\tau_{1}}^{\tau}\left[\nu\left(\frac{\theta_{y}}{v}-\frac{\Theta_{y}}{V}\right) Z g_{\beta}^{2}\right](\tau) d \tau-\int_{\tau_{1}}^{\tau} f_{\mathbf{R}} \nu\left(\frac{\theta_{y}}{v}-\frac{\Theta_{y}}{V}\right)\left(Z g_{\beta}^{2}\right)_{y} d y d \tau \\
& +\int_{\tau_{1}}^{\tau} f_{\mathbf{R}}\left(\frac{u_{y}^{2}}{v}-\frac{U_{y}^{2}}{V}\right) Z g_{\beta}^{2} d y d \tau-\int_{\tau_{1}}^{\tau} f_{\mathbf{R}} Q_{2} Z g_{\beta}^{2} d y d \tau=: \sum_{i=1}^{6} K_{i} .
\end{aligned}
$$

Here the jump term $K_{3}$ can be estimated as follows, recalling $g_{\beta}(\tau, 0) \equiv 0$.

$$
K_{3}=\int_{\tau_{1}}^{\tau}\left[\nu\left(\frac{\theta_{y}}{v}-\frac{\Theta_{y}}{V}\right) Z g_{\beta}^{2}\right](\tau) d \tau=\nu \int_{\tau_{1}}^{\tau} g_{\beta}^{2}(\tau, 0)\left(\frac{\theta_{y}}{v}-\frac{\Theta_{y}}{V}\right)(\tau, 0)[Z](\tau) d \tau \equiv 0,
$$

while the terms $K_{i}(i=1,4,5,6)$ can be directly dealt with in the same manner as in [15] or [17]. To bound the term $K_{2}$, we make use of the mass equation $(3.1)_{1}$ to write $K_{2}$ in the form

$$
\begin{aligned}
& -(p-P) \psi_{y} Z g_{\beta}^{2}=\frac{\gamma P \phi-(\gamma-1) Z}{v} Z g_{\beta}^{2} \phi_{\tau}=\frac{\gamma P Z g_{\beta}^{2}}{2 v}\left(\phi^{2}\right)_{\tau}-\frac{(\gamma-1) Z^{2} g_{\beta}^{2}}{v} \phi_{\tau} \\
& =\left(\frac{\gamma P Z \phi^{2} g_{\beta}^{2}-2(\gamma-1) \phi Z^{2} g_{\beta}^{2}}{2 v}\right)_{\tau}-\frac{\gamma P Z \phi^{2}-2(\gamma-1) Z^{2} \phi}{v} g_{\beta}\left(g_{\beta}\right)_{\tau} \\
& +\frac{\gamma P Z \phi^{2}-2(\gamma-1) Z^{2} \phi}{2 v^{2}} g_{\beta}^{2} v_{\tau}-\left(\frac{2(\gamma-1) g_{\beta}^{2} \phi Z}{v}+\frac{\gamma P g_{\beta}^{2} \phi^{2}}{2 v}\right) Z_{\tau}-\frac{\gamma g_{\beta}^{2} \phi^{2} Z}{2 v} P_{\tau},
\end{aligned}
$$

where all terms on the right-side hand of the above identity can be directly bounded in the same way as in [15] or [17]. Therefore, we have bounded $K_{2}$.

Taking $\beta=\frac{c_{0}}{2}$, one can get from Lemma 3.6 that

$$
\int_{\tau_{1}}^{\tau} f_{\mathbf{R}} \frac{e^{-\frac{c_{0} y^{2}}{1+\tau}}}{1+\tau} Z^{2} d y d \tau \leq C \delta+C\left\|(\phi, \psi, \zeta)\left(\tau_{1}, \cdot\right)\right\|^{2}+C\|(\phi, \psi, \zeta)(\tau, \cdot)\|^{2}
$$




$$
\begin{aligned}
& +C \int_{\tau_{1}}^{\tau}\left\|\left(\phi_{y}, \psi_{y}, \zeta_{y}\right) H^{2} d \tau+C \int_{\tau_{1}}^{\tau}(1+\tau)^{-\frac{7}{6}}\right\|(\phi, \psi) \|^{2} d \tau \\
& +C\left(\delta+\eta_{1}\right) \int_{\tau_{1}}^{\tau} f_{\mathbf{R}}(1+\tau)^{-1} e^{-\frac{c_{0} y^{2}}{1+\tau}}|(\phi, \zeta)|^{2} d y d \tau .
\end{aligned}
$$

Now, taking $\alpha=c_{0}$ in (3.37) and choosing $\delta$ and $\eta_{1}$ suitably small, we combine (3.37) with (3.38) to obtain the desired estimate in Lemma 3.8.

By Lemmas 3.5 and 3.8, we conclude

$$
\begin{gathered}
N\left(\tau_{1}, \tau_{2}\right)+\int_{\tau_{1}}^{\tau_{2}}\left\{\left\|\phi_{y} \#^{2}+\right\|\left(\psi_{y}, \zeta_{y}\right) \#_{1}^{2}+\left\|\left(\psi_{y \tau}, \zeta_{y \tau}\right)\right\|^{2}\right\} d \tau \\
\leq C N\left(\tau_{1}\right)+C \int_{0}^{t}(1+\tau)^{-\frac{7}{6}}\|(\phi, \psi, \zeta)\|^{2} d \tau+C \delta^{\frac{1}{4}}
\end{gathered}
$$

An application of Gronwall's inequality to the above inequality gives the estimate (3.4) in Proposition 3.3. This completes the proof of Proposition 3.1.

\section{References}

[1] S. Bianchini, A. Bressan, Vanishing viscosity solutions of nonlinear hyperbolic systems, Ann. of Math., 161 (2005), 223-342. MR2150387

[2] G. Q. Chen, M. Perepelitsa, Vanishing viscosity limit of the NavierStokes equations to the Euler equations for compressible fluid flow, Comm. Pure Appl. Math., 63, (2010), 1469-1504. MR2683391

[3] G. Q. Chen, D. Hoff, K. Trivisa, Global solutions of the compressible Navier-Stokes equations with large discontinuous initial data, Comm. Partial Diff. Eqns., 25 (2000), 2233-2257. MR1789926

[4] J. Goodman, Z. P. Xin, Viscous limits for piecewise smooth solutions to systems of conservation laws, Arch. Ration. Mech. Anal., 121 (1992), 235-265. MR1188982

[5] O. Guès, G. Métivier, M. Williams, and K. Zumbrun, Existence and stability of multidimensional shock fronts in the vanishing viscosity limit, Arch. Ration. Mech. Anal., 175 (2005), 151-244. MR2118476

[6] O. Guès, G. Métivier, M. Williams, and K. Zumbrun, Multidimensional viscous shocks II: The small viscosity limit, Comm. Pure Appl. Math., 57 (2004), 141-218. MR2012648

[7] D. Hoff, Construction of solutions for compressible, isentropic NavierStokes equations in one space dimension with nonsmooth initial data, Proc. Roy. Soc. Edinburgh (Sect. A), 103 (1986), 301-315. MR0866843 
[8] D. Hoff, Global existence for 1D, compressible, isentropic Navier-Stokes equations with large initial data, Trans. Amer. Math. Soc., 303 (1987), 169-181. MR0896014

[9] D. Hoff, Discontinuous solutions of the Navier-Stokes equations for compressible flow, Arch. Rational Mech. Anal., 114 (1991), 15-46. MR1088275

[10] D. Hoff, Global well-posedness of the Cauchy problem for the NavierStokes equations of nonisentropic flow with discontinuous initial data, J. Diff. Eqns., 95 (1992), 33-74. MR1142276

[11] D. Hoff, Discontinuous solutions of the Navier-Stokes equations for multidimensional flows of heat-conducting fluids, Arch. Rational Mech. Anal., 139 (1997), 303-354. MR1480244

[12] D. Hoff, Global solutions of the equations of one-dimensional, compressible flow with large data and forces, and with differing end states, Z. Angew. Math. Phys., 49 (1998), 774-785. MR1652200

[13] D. Hoff, T. P. Liu, The inviscid limit for the Navier-Stokes equations of compressible, isentropic flow with shock data, Indiana Univ. Math. J., 38 (1989), 861-915. MR1029681

[14] F. M. Huang, M. J. Li, and Y. Wang, Zero dissipation limit to rarefaction wave with vacuum for 1-D compressible Navier-Stokes equations, SIAM J. Math. Anal., 44 (2012), 1742-1759. MR2982730

[15] F. M. Huang, J. Li, and A. Matsumura, Asymptotic stability of combination of viscous contact wave with rarefaction waves for onedimentional compressible Navier-Stokes system, Arch. Rat. Mech. Anal., 197 (2010), 89-116. MR2646815

[16] F. M. Huang, A. Matsumura, Z. P. Xin, Stability of contact discontinuities for the 1-D compressible Navier-Stokes equations. Arch. Ration. Mech. Anal., 179 (2006), 55-77. MR2208289

[17] F. M. Huang, Y. Wang, and T. Yang, Fluid dynamic limit to the Riemann solutions of Euler equations: I. Superposition of rarefaction waves and contact discontinuity, Kinetic and Related Models, 3 (2010), 685728. MR2735911

[18] F. M. Huang, Y. Wang, and T. Yang, Vanishing viscosity limit of the compressible Navier-Stokes equations for solutions to Riemann problem, Arch. Ration. Mech. Anal., 203 (2012) 379-413. MR2885565 
[19] F. M. Huang, Y. Wang, Y. Wang, T. Yang, The limit of the Boltzmann equation to the Euler equations for Riemann problems, SIAM J. Math. Anal., 45 (2013), 1741-1811.

[20] S. Jiang, G. X. Ni, and W. J. Sun, Vanishing viscosity limit to rarefaction waves for the Navier-Stokes equations of one-dimensional compressible heat-conducting fluids, SIAM J. Math. Anal., 38 (2006), 368-384. MR2237152

[21] S. X. Ma, Zero dissipation limit to strong contact discontinuity for the 1-D compressible Navier-Stokes equations, J. Diff. Eqns., 248 (2010), 95-110. MR2557896

[22] A. Matsumura, K. Nishihara, Asymptotics toward the rarefaction wave of the solutions of a one-dimensional model system for compressible viscous gas, Japan J. Appl. Math., 3 (1986), 1-13. MR0899210

[23] X. Qin, Y. Wang, Stability of wave patterns to the inflow problem of full compressible Navier-Stokes equations, SIAM J. Math. Anal., 41 (2009), 2057-2087. MR2578799

[24] X. Qin, Y. Wang, Large-time behavior of solutions to the inflow problem of full compressible Navier-Stokes equations. SIAM J. Math. Anal., 43 (2011), 341-366. MR2765694

[25] J. Smoller, Shock Waves and Reaction-Diffusion Equations, 2nd ed., New York: Springer-Verlag, xxii, 1994. MR1301779

[26] H. Y. Wang, Viscous limits for piecewise smooth solutions of the psystem, J. Math. Anal. Appl., 299 (2004), 411-432. MR2098251

[27] Y. Wang, Zero dissipation limit of the compressible heat-conducting Navier-Stokes equations in the presence of the shock, Acta Mathematica Scientia, 28B (2008), 727-748. MR2462917

[28] Z. P. Xin, Zero dissipation limit to rarefaction waves for the onedimensional Navier-Stokes equations of compressible isentropic gases, Comm. Pure Appl. Math., 46 (1993), 621-665. MR1213990

[29] Z. P. Xin, H. H. Zeng, Convergence to the rarefaction waves for the nonlinear Boltzmann equation and compressible Navier-Stokes equations, J. Diff. Eqns., 249 (2010), 827-871. MR2652155

[30] S. H. Yu, Zero-dissipation limit of solutions with shocks for systems of hyperbolic conservation laws, Arch. Ration. Mech. Anal., 146 (1999), 275-370. MR1718368 
[31] Y. H. Zhang, R. H. Pan, Y. Wang, and Z. Tan, Zero dissipation limit with two interacting shocks of the 1D non-isentropic Navier-Stokes equations, Indiana Univ. Math. J., 62 (2013), 249-309. MR3158509

FEImin HuAng

Institute of ApPlied Mathematics

AMSS, CAS

BEIJING 100190

P.R. CHINA

E-mail address: fhuang@amt.ac.cn

SONG JIANG

Institute of Applied Physics and Computational Mathematics

BEIJING 100088

CHINA

E-mail address: jiang@iapcm.ac.cn

YI WANG

Institute of Applied Mathematics

AMSS, CAS

BEIJING 100190

P.R. CHINA

E-mail address: wangyi@amss.ac.cn

Received August 29, 2013 